\title{
Water-Dispersible Copper Sulfide Nanocrystals via Ligand Exchange of 1-Dodecanethiol
}

Christina H. M. van Oversteeg, ${ }^{\dagger, \oplus \odot}$ Freddy E. Oropeza, ${ }^{\S}$ Jan P. Hofmann, ${ }^{\S} \odot$ Emiel J. M. Hensen, ${ }^{\S \odot}$ Petra E. de Jongh, ${ }^{\ddagger \oplus}$ and Celso de Mello Donega ${ }^{*}, \oplus \odot$

${ }^{\dagger}$ Condensed Matter and Interfaces, Debye Institute for Nanomaterials Science, Utrecht University, 3508 TA Utrecht, The Netherlands

${ }^{\ddagger}$ Inorganic Chemistry and Catalysis, Debye Institute for Nanomaterials Science, Utrecht University, 3508 TA Utrecht, The Netherlands

${ }^{\S}$ Laboratory of Inorganic Materials Chemistry, Department of Chemical Engineering and Chemistry, Eindhoven University of Technology, Postbox 513, 5600 MB Eindhoven, The Netherlands

\section{Supporting Information}

ABSTRACT: In colloidal $\mathrm{Cu}_{2-x} \mathrm{~S}$ nanocrystal synthesis, thiols are often used as organic ligands and the sulfur source, as they yield high-quality nanocrystals. However, thiol ligands on $\mathrm{Cu}_{2-x} \mathrm{~S}$ nanocrystals are difficult to exchange, limiting the applications of these nanocrystals in photovoltaics, biomedical sensing, and photocatalysis. Here, we present an effective and facile procedure to exchange native 1-dodecanethiol on $\mathrm{Cu}_{2-x} \mathrm{~S}$ nanocrystals by 3-mercaptopropionate, 11-mercaptoundecanoate, and $\mathrm{S}^{2-}$ in formamide under inert atmosphere. The product hydrophilic $\mathrm{Cu}_{2-x} \mathrm{~S}$ nanocrystals have excellent colloidal stability in formamide. Furthermore, the size, shape, and optical properties of the nanocrystals are not significantly affected by the ligand exchange. Water-dispersible $\mathrm{Cu}_{2-x} \mathrm{~S}$ nanocrystals are easily obtained by precipitation of the nanocrystals capped by $\mathrm{S}^{2-}$, 3-mercaptopropionate, or 11-mercaptoundecanoate from formamide, followed by redispersion in water. Interestingly, the ligand exchange rates for $\mathrm{Cu}_{2-x} \mathrm{~S}$ nanocrystals capped with 1-dodecanethiol are observed to depend on the preparation method, being much slower for $\mathrm{Cu}_{2-x} \mathrm{~S}$ nanocrystals prepared through heating-up than through hot-injection synthesis protocols. XPS studies reveal that the differences in the ligand exchange rates are due to the surface chemistry of the $\mathrm{Cu}_{2-x} \mathrm{~S}$ nanocrystals, where the nanocrystals prepared via hot-injection synthesis have a less dense ligand layer due to the presence of trioctylphosphine oxide during synthesis. A model is proposed that explains the observed differences in the ligand exchange rates. The facile ligand exchange procedures reported here enable the use of high-quality colloidal Cu $\mathrm{Cu}_{2-x} \mathrm{~S}$ nanocrystals prepared in the presence of 1 -dodecanethiol in various applications.

\section{INTRODUCTION}

Copper sulfide $\left(\mathrm{Cu}_{2-x} \mathrm{~S}\right)$ nanocrystals have attracted increasing attention in the past decade for their use in various applications, such as biomedical sensing, ${ }^{1-3}$ photothermal therapy, $^{2-4}$ photovoltaic and plasmonic devices, ${ }^{2,5-8}$ and photo- and electrocatalysis. ${ }^{2,5,9,10} \mathrm{Cu}_{2-x} \mathrm{~S}$ can accommodate copper vacancies and hence can exist in a variety of compositions and crystal structures. ${ }^{11}$ The interest in $\mathrm{Cu}_{2-x} \mathrm{~S}$ can be understood from its unique optoelectronic properties that depend on the size, shape, and composition of the nanocrystals. The material is a p-type semiconductor that has a stoichiometry dependent, direct band gap ranging from $1.2 \mathrm{eV}$ for chalcocite $\left(\mathrm{Cu}_{2} \mathrm{~S}\right)$ up to $2.0 \mathrm{eV}$ for covellite $(\mathrm{CuS}) .{ }^{11-13}$ Furthermore, depending on the composition, $\mathrm{Cu}_{2-x} \mathrm{~S}$ nanocrystals show a broad absorption in the near-infrared spectral region. This absorption is related to localized surface plasmon resonances (LSPR) of the holes in the top of the valence band, which are introduced in the material by copper vacancies. ${ }^{5-7,12}$ In addition, $\mathrm{Cu}_{2-x} \mathrm{~S}$ is of interest for various applications due to its abundance and low toxicity. $5,11,14$

Due to the dependence of the optical and electronic properties on nanocrystal size, shape, and composition, the preparation of monodisperse samples with uniform properties is of great interest. Colloidal synthesis methods are known to allow for a high degree of control over the size, shape, and composition of the nanocrystals. ${ }^{15}$ For example, nanoplatelets, nanosheets, nanodisks, and spherical $\mathrm{Cu}_{2-x} \mathrm{~S}$ nanocrystals have been synthesized with narrow size and shape distributions. ${ }^{16-22}$ In colloidal synthesis, nanocrystals are grown in solution by chemical precipitation reactions of copper and

Received: November 2, 2018

Revised: December 18, 2018

Published: December 19, 2018 
sulfur precursors in the presence of organic ligands. The best known colloidal synthesis technique is hot-injection, in which the precursors are rapidly injected into a preheated reaction mixture containing the other reagents. The rapid injection leads to a high availability of monomers and hence a burst of nucleation, followed by further growth of the existing nuclei. Although hot-injection synthesis protocols are well-developed and can give nanocrystals with a large range of sizes, shapes, and compositions, they usually only yield a small amount of product and cannot be easily scaled up, as factors like injection rate and mass and heat transport become less reproducible for larger reaction volumes. ${ }^{23}$ An alternative to the hot-injection method is the so-called heating-up method, where all reagents are mixed in a reaction flask and heated to induce nucleation and growth of the nanocrystals. This method allows for a more reproducible and scalable synthesis, since factors such as injection rate and nonideal mixing do not play a role. ${ }^{23}$

Organic ligands are important during the synthesis as they allow for the high control over size, shape, and composition of the nanocrystals by dynamically binding to the nanocrystal surface. ${ }^{15}$ Furthermore, after synthesis they prevent aggregation of the nanocrystals by steric stabilization. These ligands usually have a polar headgroup coordinating to the nanocrystal surface atoms through a donor atom (e.g., S, N, O) and a long apolar hydrocarbon tail that stabilizes the nanocrystals in apolar solvents. ${ }^{15}$ Commonly used ligands include phosphines, amines, and thiols. Though ligands are essential during and after synthesis, they lead to challenges when using the nanocrystals in applications. The apolar nature of the hydrocarbon chain prevents dispersion of the nanocrystals in water and other polar solvents, limiting their use in, e.g., biomedical and photocatalytic applications. Furthermore, the bulky organic ligands can restrict interparticle conductivity and can render the nanocrystal surface inaccessible to reactants, hindering the use of the nanocrystals in optoelectronic, photovoltaic, and photo- and electrocatalytic applications. As a consequence, ligand exchange is crucial to obtain colloidal nanocrystals suitable for a broad range of applications. Various procedures have been reported for ligand exchange on colloidal nanocrystals. For example, Nag et al. reported the preparation of all-inorganic nanocrystals by replacing native organic ligands with inorganic ions such as $\mathrm{S}^{2-}, \mathrm{HS}^{-}$, and $\mathrm{OH}^{-24}$ Kovalenko et al. described the stabilization of colloidal nanocrystals in polar solvents by using molecular metal chalcogenide ligands, such as $\mathrm{Sn}_{2} \mathrm{~S}_{6}{ }^{4-} \cdot{ }^{25,26}$ By a subsequent heating step, the ligands could be converted into semiconducting phases, realizing conductive arrays of nanoparticles. Aqueous phase transfers have also been extensively reported using organic ligands such as 3-mercaptopropionic acid (MPA), ${ }^{27-33} 11$-mercaptoundecanoic acid (MUA), ${ }^{31-35}$ cysteine, $^{36}$ and various polymers. $^{37-39}$

In $\mathrm{Cu}_{2-x} \mathrm{~S}$ nanocrystal synthesis, thiols are often used as ligands as they give high quality, monodisperse products. ${ }^{40,41}$ However, no effective ligand exchange procedures have been reported for $\mathrm{Cu}_{2-x} \mathrm{~S}$ and $\mathrm{CuInS}_{2}$ nanocrystals prepared via direct synthesis in the presence of thiols. ${ }^{40,42-47} \mathrm{Xie}$ et al. reported the resistance of $\mathrm{CuInS}_{2}$ nanocrystals obtained with thiol ligands toward a ligand exchange using MPA. ${ }^{42}$ Turo et al. described the resistance of $\mathrm{Cu}_{2-x} \mathrm{~S}$ nanocrystals prepared with 1-dodecanethiol (DDT) toward ligand exchange procedures and attributed this to the presence of so-called crystal-bound thiols, where the thiols occupy high coordination number sites and are thus strongly bound to the nanocrystal. ${ }^{40}$
A similar explanation has been given by other groups. ${ }^{43-45}$ More recently, Gromova et al. reported on the difficulty of surface functionalization of $\mathrm{CuInS}_{2}$ nanocrystals prepared in the presence of DDT. They attributed this to the presence of a ligand double layer on the nanocrystals surface, consisting of dodecanethiolate ligands and thioether species formed in situ during the heating-up synthesis in the presence of thiols. ${ }^{46}$

In this work, we report on ligand exchange procedures for $\mathrm{Cu}_{2-x} \mathrm{~S}$ nanocrystals prepared by hot-injection and heating-up synthesis protocols in the presence of DDT. In contrast to the studies mentioned above, ${ }^{40,42-47}$ we developed procedures to replace $\mathrm{DDT}$ on $\mathrm{Cu}_{2-x} \mathrm{~S}$ nanocrystals with $\mathrm{MPA}^{-}, \mathrm{MUA}^{-}$, or $\mathrm{S}^{2-}$ by performing the ligand exchange procedures under inert atmosphere in a highly polar solvent (formamide). In addition, we found that the ligand exchange rates are highly dependent on both the nature of the replacing ligands $\left(\mathrm{MPA}^{-}, \mathrm{MUA}^{-}\right.$, or $\mathrm{S}^{2-}$ ) and on the synthesis method used to produce the $\mathrm{Cu}_{2-x} \mathrm{~S}$ nanocrystals (viz., hot-injection or heating-up). XPS studies revealed that the differences observed between the ligand exchange rates involving nanocrystals prepared by the different synthesis protocols are due to the surface chemistry of the nanocrystals, where those synthesized by the heating-up protocol have a denser native thiolate ligand layer, in comparison with those prepared by the hot-injection method in the presence of trioctylphosphine oxide (TOPO). Finally, we propose a model for ligand exchange on thiolate capped $\mathrm{Cu}_{2-x} \mathrm{~S}$ nanocrystals that explains the observed differences in the ligand exchange rates. The ligand exchange protocols developed in this work present a facile way to achieve waterdispersible $\mathrm{Cu}_{2-x} \mathrm{~S}$ nanocrystals with, depending on the replacing ligand, accessible nanocrystal surfaces.

\section{RESULTS AND DISCUSSION}

Ligand Exchange Procedures on $\mathrm{Cu}_{2 x} \mathrm{~S}$ Nanocrystals Prepared with Thiols. $\mathrm{Cu}_{2-x} \mathrm{~S}$ nanocrystals were synthesized according to two previously described protocols, where DDT was used as both sulfur source and ligand (see Experimental Section for details). In the first method, $\mathrm{Cu}_{2-x} \mathrm{~S}$ nanocrystals were synthesized using a hot-injection technique. DDT was injected into a mixture of $\mathrm{Cu}(\mathrm{I})$ acetate, trioctylphosphineoxide (TOPO), and 1-octadecene (ODE). ${ }^{34,48}$ During this synthesis, both TOPO and DDT ligands direct the growth of the nanocrystals. ${ }^{48}$ These nanocrystals will hereafter be referred to as HI_DDT/TOPO. $\mathrm{Cu}_{2-x} \mathrm{~S}$ nanocrystals were also synthesized by heating $\mathrm{CuSO}_{4} \cdot 5 \mathrm{H}_{2} \mathrm{O}, \mathrm{DDT}$, and oleic acid (OA) to $200{ }^{\circ} \mathrm{C}$ in a so-called heating-up synthesis. ${ }^{49}$ These nanocrystals will hereafter be referred to as HU DDT.

To replace the native ligands on the $\overline{\mathrm{C}} \mathrm{u}_{2-x} \mathrm{~S}$ nanocrystals and obtain water-dispersible $\mathrm{Cu}_{2-x} \mathrm{~S}$ nanocrystals, ligand exchange procedures using MPA, MUA, and $\mathrm{S}^{2-}$ (either from $\left(\mathrm{NH}_{4}\right)_{2} \mathrm{~S}$ or $\mathrm{Na}_{2} \mathrm{~S}$ ) were studied. In a typical ligand exchange reaction a two-phase system was formed by combining the $\mathrm{Cu}_{2-x} \mathrm{~S}$ nanocrystals in an apolar solvent (e.g., toluene) with an excess of the replacing ligand (MPA, MUA, or $\mathrm{S}^{2-}$ ) in polar solvent (e.g., water or formamide). The $\mathrm{pH}$ of the solutions containing MUA was increased to 11 using tetramethylammonium hydroxide (TMAH) in order to deprotonate the carboxylic acid group, thereby rendering the MUA water-soluble. The solutions containing $\mathrm{S}^{2-}$ were also alkaline as the $\mathrm{S}^{2-}$ undergoes hydrolysis, forming $\mathrm{HS}^{-}$and $\mathrm{OH}^{-}$. In contrast, the MPA solution has a low $\mathrm{pH}$ of 3 at the start of the ligand exchange experiments. Although not needed for the solubility of MPA in formamide or water, experiments using MPA were 

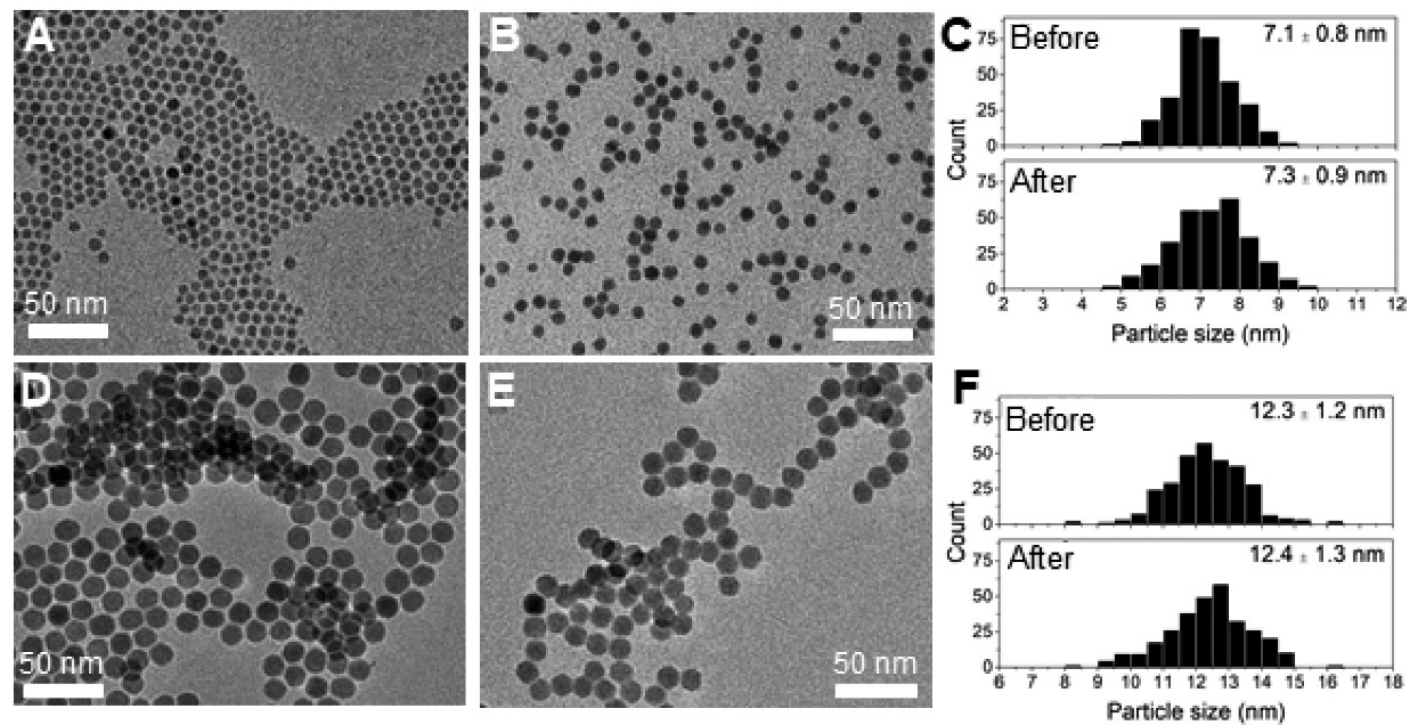

Figure 1. TEM images of HI_DDT/TOPO (A) before and (B) after ligand exchange with $\mathrm{S}^{2-}$ and (C) corresponding particle size histograms. TEM images of HU_DDT (D) before and (E) after ligand exchange with $\mathrm{S}^{2-}$ and $(\mathrm{F})$ corresponding particle size histograms.

also performed at $\mathrm{pH} 11$ to compare the different replacing ligands under identical conditions. No differences in phase transfer rate were observed between the MPA experiments carried out at low and those carried out at high pHs. Therefore, for the sake of conciseness, only experiments using MPA at low $\mathrm{pH}$ are further discussed.

The success of the ligand exchange was evidenced by transfer of the $\mathrm{Cu}_{2-x} \mathrm{~S}$ nanocrystals from the apolar to the polar phase (Supporting Information, Figure S1). Different experimental conditions were studied for the ligand exchange experiments. First, experiments were performed in air using water as the polar solvent. Subsequently, experiments were performed in air, using formamide as the polar solvent. Lastly, experiments were performed under air-free conditions and using formamide as the polar solvent. The three different sets of experiments are discussed in more detail below.

At first, ligand exchange procedures were performed using water in air, as described elsewhere. ${ }^{2,34}$ Using this procedure, ligand exchange using $\mathrm{MPA}^{-}$and $\mathrm{S}^{2-}$ (either from $\left(\mathrm{NH}_{4}\right)_{2} \mathrm{~S}$ or $\mathrm{Na}_{2} \mathrm{~S}$ ) as the replacing ligands was successful. However, the colloidal stability of the product nanocrystals was low, and aggregates formed during the phase transfer process. In addition, the sample obtained using $\left(\mathrm{NH}_{4}\right)_{2} \mathrm{~S}$ turned from black to blue after several hours, suggesting the formation of $\mathrm{Cu}^{2+}$-ammonia complexes. Copper has a valency of +1 in the $\mathrm{Cu}_{2-x} \mathrm{~S}$ nanocrystals, but in the presence of oxygen, part of the $\mathrm{Cu}^{+}$ions may be oxidized to $\mathrm{Cu}^{2+} \cdot{ }^{40,50}$ In aqueous solution, the ammonium ion can dissociate into ammonia and a proton through $\mathrm{NH}_{4}^{+}(\mathrm{aq}) \rightleftharpoons \mathrm{NH}_{3}(\mathrm{aq})+\mathrm{H}^{+}(\mathrm{aq})$. In the presence of $\mathrm{NH}_{3}(\mathrm{aq}), \mathrm{Cu}^{2+}$ can then form the stable $\left[\mathrm{Cu}\left(\mathrm{NH}_{3}\right)_{4}\right]^{2+}$ complex that has an intense violet-blue color. ${ }^{51,52}$ Due to the instability of the $\mathrm{Cu}_{2-x} \mathrm{~S}$ nanocrystals in the presence of $\mathrm{NH}_{3}$ / $\mathrm{NH}_{4}^{+},\left(\mathrm{NH}_{4}\right)_{2} \mathrm{~S}$ was not used as the $\mathrm{S}^{2-}$ source in further experiments. When using $\mathrm{S}^{2-}$ as the replacing ligand, both the HI_DDT/TOPO and the HU_DDT nanocrystals were transferred to the polar water phase upon stirring for about $5 \mathrm{~h}$. When MPA was used as the replacing ligand, both the HI DDT/TOPO and the HU DDT nanocrystals were transferred to the polar aqueous MPA solution upon stirring overnight. In contrast, when MUA was used as the replacing ligand, only partial transfer of the HI_DDT/TOPO nanocrystals was observed upon stirring overnight, while for the HU_DDT nanocrystals no phase transfer was observed, even after stirring for 3 days.

As the polarity of the solvent could influence the success of the ligand exchange by stabilizing charged intermediates, the ligand exchange was also performed using formamide as the polar solvent under ambient conditions. Formamide has a higher polarity $(\varepsilon=106)$ than water $(\varepsilon=80) .{ }^{24}$ However, upon stirring for several hours, the color of the MUA and $\mathrm{Na}_{2} \mathrm{~S}$ solution layers changed from colorless to black and then to violet-blue, while the apolar phase initially containing the $\mathrm{Cu}_{2-x} \mathrm{~S}$ nanocrystals turned from black to colorless. This indicates that the $\mathrm{Cu}_{2-x} \mathrm{~S}$ nanocrystals were successfully transferred to the polar phase but were not stable after the transfer. The dark blue color again suggests the formation of $\mathrm{Cu}^{2+}$ complexes, where formamide itself can act as the ligand. Complex formation between $\mathrm{Cu}^{2+}$ and formamide has been observed in oxidative and alkaline environments, ${ }^{53}$ where $\mathrm{Cu}^{2+}$ was leaking into solution from either $\mathrm{Cu}_{2} \mathrm{~S}^{54}$ or copper foil ${ }^{55,56}$ and was quickly coordinated by formamide. The blue color was also observed when using MPA, but only after storing the MPA-capped $\mathrm{Cu}_{2-x} \mathrm{~S}$ nanocrystals in formamide for several days in air. The alkaline nature of the solutions containing MUA or $\mathrm{Na}_{2} \mathrm{~S}$ when compared to the more acidic MPA solution when initiating the ligand exchange experiments can possibly explain the slower complex formation between $\mathrm{Cu}^{2+}$ and formamide observed in MPA solutions. ${ }^{54}$

To prevent the oxidation of $\mathrm{Cu}^{+}$to $\mathrm{Cu}^{2+}$ and the rapid complexation of $\mathrm{Cu}^{2+}$ by formamide, the ligand exchange experiments using formamide were performed under inert atmosphere in a nitrogen glovebox $\left(\leq 6 \mathrm{ppm}\right.$ of $\left.\mathrm{O}_{2}\right)$. We found that under these conditions the native ligands on the $\mathrm{Cu}_{2-x} \mathrm{~S}$ nanocrystals could successfully be replaced with all three ligands $\left(\mathrm{MPA}^{-}, \mathrm{MUA}^{-}\right.$, and $\mathrm{S}^{2-}$ from $\mathrm{Na}_{2} \mathrm{~S}$ ). Colloidal stability in the polar formamide was obtained by binding the negatively charged $\mathrm{MPA}^{-}, \mathrm{MUA}^{-}$, and $\mathrm{S}^{2-}$ to the nanocrystal surface. The negative charge of the ligands was confirmed with $\zeta$-potential measurements. $\mathrm{Cu}_{2-x} \mathrm{~S}$ nanocrystals prepared by hot-injection synthesis gave $\zeta$-potentials of $-44.4 \mathrm{mV},-31.1 \mathrm{mV}$, and -34.9 
$\mathrm{mV}$ for nanocrystals capped with $\mathrm{S}^{2-}, \mathrm{MPA}^{-}$, and $\mathrm{MUA}^{-}$, respectively (Supporting Information, Figure S2). For the $\mathrm{Cu}_{2-x} \mathrm{~S}$ nanocrystals prepared by heating-up synthesis, the $\zeta$ potentials were $-31.3 \mathrm{mV},-33.9 \mathrm{mV}$, and $-34.0 \mathrm{mV}$ for nanocrystals capped with $\mathrm{S}^{2-}, \mathrm{MPA}^{-}$, and $\mathrm{MUA}^{-}$, respectively (Supporting Information, Figure S2). The resulting hydrophilic nanocrystals have a high colloidal stability and were stored in formamide inside the glovebox for several months without losing colloidal stability. To obtain water-dispersible $\mathrm{Cu}_{2-x} \mathrm{~S}$ nanocrystals, the particles were precipitated using acetonitrile as antisolvent and isolated by centrifugation, followed by redispersion in water. When dispersed in water, slight turbidity developed over time (on a time scale of $10 \mathrm{~min}$ for $\mathrm{S}^{2-}$ capped nanocrystals and days for $\mathrm{MPA}^{-}$and $\mathrm{MUA}^{-}$ capped nanocrystals), indicating that the colloidal stability of the hydrophilic $\mathrm{Cu}_{2-x} \mathrm{~S}$ nanocrystals is lower in water than in formamide. This difference in colloidal stability is ascribed to the polarity of the solvent, where the charged nanocrystals are better stabilized in the highly polar formamide $(\varepsilon=106)$ than in the less polar water $(\varepsilon=80) .^{24}$

Impact of Ligand Exchange on Nanocrystal Size, Crystal Structure, and Optical Properties. Figure 1 shows transmission electron microscopy (TEM) images of the $\mathrm{Cu}_{2-x} \mathrm{~S}$ nanocrystals before (Figure 1A,D) and after (Figure $1 \mathrm{~B}, \mathrm{E})$ ligand exchange with $\mathrm{S}^{2-}$ in formamide under an inert atmosphere. The corresponding particle size histograms (Figure 1C,F) show the size of the $\mathrm{Cu}_{2-x} \mathrm{~S}$ nanocrystals without the ligand layer. Before ligand exchange, a particle size of $7.1 \pm 0.8 \mathrm{~nm}$ was found for the nanocrystals prepared by hot-injection synthesis, whereas the nanocrystals prepared by heating-up synthesis had a size of $12.3 \pm 1.2 \mathrm{~nm}$. TEM analysis shows that the size and shape of the nanocrystals were not significantly affected by exchanging the native DDT ligands by any of the replacing ligands (see Figure 1 for $\mathrm{S}^{2-}$, and Supporting Information, Figure S3, for MPA and MUA).

In addition, the TEM images show that the nanocrystals assembled in organized arrays when dried on the TEM grid (Figure 1). The separation between the nanocrystals in such an array can be related to the organic ligands capping the nanocrystals. The interparticle distance was $1.7 \pm 0.2 \mathrm{~nm}$ for both the as-synthesized sample prepared by hot-injection in the presence of TOPO and by heating-up synthesis without TOPO. This distance is very similar to the DDT chain length (viz., $1.8 \mathrm{~nm}) .{ }^{57}$ Upon ligand exchange with $\mathrm{S}^{2-}$ the nanocrystals come in very close proximity to each other (Figure 1B,E). This can be explained by the replacement of 1dodecanethiol by the smaller $\mathrm{S}^{2-}$.

The crystal structure of the $\mathrm{Cu}_{2-x} \mathrm{~S}$ nanocrystals before and after ligand exchange was investigated using X-ray diffraction (XRD) (Figure 2A). Prior to ligand exchange, the nanocrystals prepared by both hot-injection and heating-up synthesis can be identified as chalcocite $\left(\mathrm{Cu}_{1.997-2.0} \mathrm{~S}\right)$ or djurleite $\left(\mathrm{Cu}_{1.94} \mathrm{~S}\right)$. Based on the XRD patterns, no distinction can be made between these two phases. However, as the copper-deficient djurleite phase is more thermodynamically stable due to its lower crystallographic symmetry compared to the chalcocite phase, the nanocrystals are likely in the djurleite phase. ${ }^{8}$ After ligand exchange with $\mathrm{S}^{2-}$ and phase transfer to formamide, the $\mathrm{Cu}_{2-x} \mathrm{~S}$ nanocrystals remain in the djurleite phase (Supporting Information, Figure S4). However, after phase transfer to water and exposure to air the nanocrystals partially oxidized to a roxbyite $\left(\mathrm{Cu}_{1.81} \mathrm{~S}\right)$ phase (Figure 2$)$. This can be explained by the formation of copper vacancies under oxygen. In this phase,
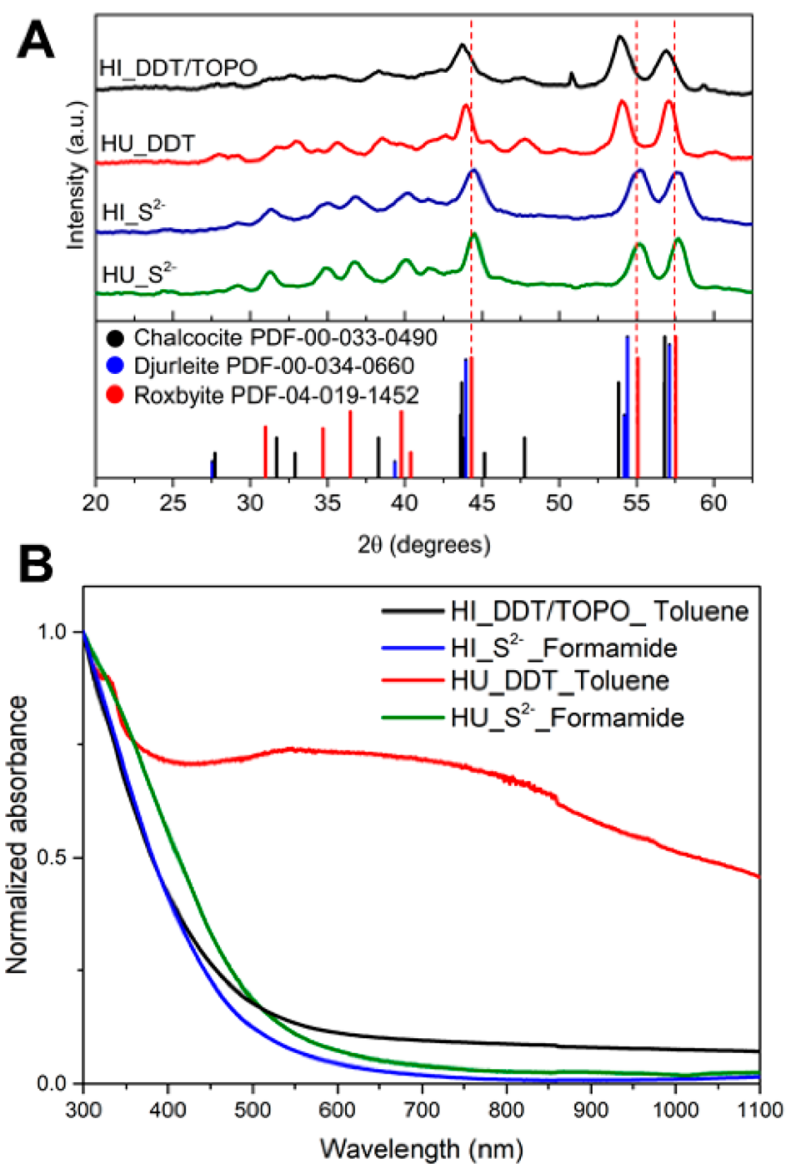

Figure 2. (A) X-ray diffractograms of the $\mathrm{Cu}_{2-x} \mathrm{~S}$ nanocrystals before (HI_DDT/TOPO and HU_DDT) and after ligand exchange

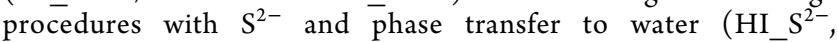
HU $\left.\mathrm{S}^{2-}\right)$. The dashed lines mark the position of the roxbyite peaks. (B) Absorption spectra of $\mathrm{Cu}_{2-x} \mathrm{~S}$ nanocrystals before (HI_DDT/TOPO and HU_DDT) and after ligand exchange with $S^{2-}$.

the copper atoms in the $\mathrm{Cu}_{2-x} \mathrm{~S}$ nanocrystal remain in the +1 oxidation state, whereas the formal valency of sulfur partially evolves from -2 to $-1 .^{58}$ This partial oxidation was observed for both the samples prepared by hot-injection and by heatingup synthesis when replacing DDT with MUA or MPA using water as the polar solvent (Supporting Information, Figure S5).

The absorption spectra of the $\mathrm{Cu}_{2-x} \mathrm{~S}$ nanocrystals before and after ligand exchange with $\mathrm{S}^{2-}$ are shown in Figure 2B. Colloidal $\mathrm{Cu}_{2-x} \mathrm{~S}$ nanocrystals typically show a broad and featureless absorption with an onset in the visible region, accompanied by a lower energy tail, ${ }^{6,8,13}$ which can be ascribed to the presence of excess holes in the valence band (the socalled Urbach tail). ${ }^{13}$ Excess holes (i.e., p-doping) are commonly observed in $\mathrm{Cu}$-chalcogenide nanocrystals due to $\mathrm{Cu}$-vacancies and also give rise to a broad absorption band in the NIR due to localized surface plasmon resonances (LSPR) that emerge when the density of holes is sufficiently large (i.e., when the concentration of $\mathrm{Cu}$-vacancies is sufficiently high). ${ }^{6,8,13,59}$ The absorption spectra of the $\mathrm{Cu}_{2-x} \mathrm{~S}$ nanocrystals prepared by hot-injection indeed show a clear absorption onset due to the band-edge absorption transition and a weak tail toward longer wavelengths. The absorption in the NIR is negligible, showing that LSPR bands are not present. These observations imply that the concentration of $\mathrm{Cu}$-vacancies is 
very low, consistent with the observed crystal structure (Figure $2 \mathrm{~A})$. The absorption spectrum after ligand exchange with $\mathrm{S}^{2-}$ is essentially unchanged, indicating that the optical properties of the $\mathrm{Cu}_{2-x} \mathrm{~S}$ nanocrystals are not affected by the ligand exchange. The absorption spectrum of the $\mathrm{Cu}_{2-x} \mathrm{~S}$ nanocrystals prepared by heating-up is quite different, with a broad feature between 500 and $1000 \mathrm{~nm}$ that is not observed in the samples prepared by hot-injection. This feature cannot be ascribed to an LSPR band because it occurs at too short wavelengths. In addition, the spectral position of this feature shifts after the sample is placed in a sonic bath. Hence, we ascribe this spectral feature to light scattering due to the presence of nanocrystal aggregates. ${ }^{6}$ Importantly, upon ligand exchange with $\mathrm{S}^{2-}$, this feature is no longer observed in the absorption spectrum, which shows instead the characteristic absorption of nearly stoichiometric $\mathrm{Cu}_{2-x} \mathrm{~S}$ nanocrystals, being very similar to that of the nanocrystals synthesized by hot-injection (Figure 2B). This is consistent with the fact that no aggregates are observed after the ligand exchange procedure in either of the samples. Similar spectra are obtained upon exchange with $\mathrm{MUA}^{-}$and $\mathrm{MPA}^{-}$(Supporting Information, Figure S6). These observations confirm that the optical properties of the $\mathrm{Cu}_{2-x} \mathrm{~S}$ nanocrystals are preserved upon ligand exchange and phase transfer.

Dependence of the Ligand Exchange Rates on the Native and Replacing Ligand and Synthesis Method. The difficulty of ligand exchange on $\mathrm{Cu}_{2-x} \mathrm{~S}$ nanocrystals has been ascribed to the presence of thiolate ligands at the nanocrystal surface. ${ }^{40,46}$ To verify whether other ligands are easier to replace, the ligand exchange procedures used for DDT-capped $\mathrm{Cu}_{2-x} \mathrm{~S}$ nanocrystals were also performed on $\mathrm{Cu}_{2-x} \mathrm{~S}$ nanocrystals capped by oleylamine. Oleylamine is expected to bind weakly to the $\mathrm{Cu}_{2-x} \mathrm{~S}$ nanocrystal surface, because it is a relatively hard Lewis base and consequently will not have a strong interaction with the soft Lewis acid $\mathrm{Cu}^{+}$in the $\mathrm{Cu}_{2-x} \mathrm{~S}$ nanocrystal. First, $\mathrm{Cu}_{2-x} \mathrm{~S}$ nanocrystals of $\sim 7 \mathrm{~nm}$ capped with oleylamine ligands were synthesized (Figure 3A). Similar to the $\mathrm{Cu}_{2-x} \mathrm{~S}$ nanocrystals prepared with DDT, the obtained nanocrystals were in a djurleite $\left(\mathrm{Cu}_{1.94} \mathrm{~S}\right)$ crystal phase (Figure 3B).

As expected from their lower binding strength, oleylamine ligands on the $\mathrm{Cu}_{2-x} \mathrm{~S}$ nanocrystals were rapidly replaced by $\mathrm{MPA}^{-}, \mathrm{MUA}^{-}$, and $\mathrm{S}^{2-}$ using the methods described above, leading to much faster phase transfer than observed for the DDT-capped $\mathrm{Cu}_{2-x} \mathrm{~S}$ nanocrystals. For example, when replacing oleylamine for $\mathrm{S}^{2-}$ using $\mathrm{Na}_{2} \mathrm{~S}$ in formamide under inert atmosphere, phase transfer of the $\mathrm{Cu}_{2-x} \mathrm{~S}$ nanocrystals from toluene to formamide occurred within minutes, while it took about $1 \mathrm{~h}$ for DDT-capped $\mathrm{Cu}_{2-x} \mathrm{~S}$ nanocrystals under the same conditions. However, whereas the size and shape of the nanocrystals were well preserved in ligand exchange procedures using $\mathrm{S}^{2-}$ as the replacing ligand on DDT-capped $\mathrm{Cu}_{2-x} \mathrm{~S}$ nanocrystals (see Figure 1 above), the product nanocrystals from the oleylamine-capped $\mathrm{Cu}_{2-x} \mathrm{~S}$ nanocrystals were highly aggregated (Figure $3 \mathrm{C}$ ). A possible explanation for this observation is the fast rate of ligand exchange for the oleylamine-capped $\mathrm{Cu}_{2-x} \mathrm{~S}$ nanocrystals. The fast stripping of the native ligands during the exchange could lead to an abrupt destabilization of the nanocrystals if the incoming $\mathrm{S}^{2-}$ ligands cannot provide sufficient charge stabilization of the nanocrystals fast enough, causing the nanocrystals to aggregate while at the interface between the two phases. This hypothesis is supported by the observation that when the bulkier MUA is
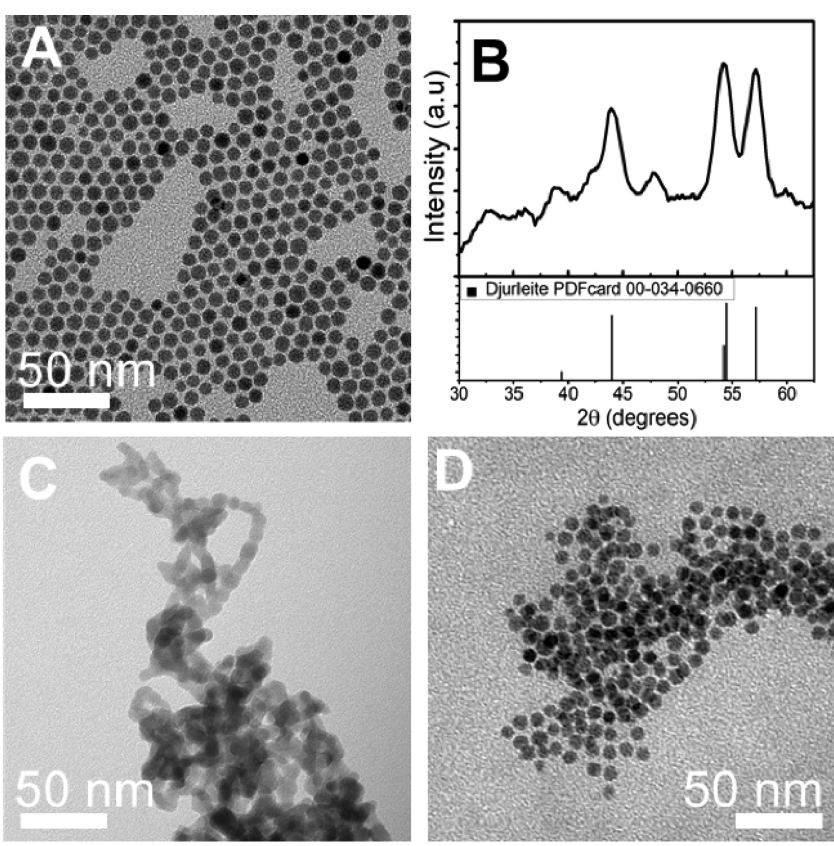

Figure 3. (A) TEM image and (B) X-ray diffractogram of $\mathrm{Cu}_{2-x} \mathrm{~S}$ nanocrystals as-synthesized capped with oleylamine. TEM images of the oleylamine capped $\mathrm{Cu}_{2-x} \mathrm{~S}$ nanocrystals after ligand exchange with (C) $\mathrm{S}^{2-}$ and (D) MUA.

used as the replacing ligand on the oleylamine-capped nanocrystals, the phase transfer was slower (on the order of hours) than when using $\mathrm{S}^{2-}$ (on the order of minutes) and the product nanocrystals did preserve their size and shape, with negligible aggregation (Figure 3D). The negligible aggregation of the $\mathrm{MUA}^{-}$-capped $\mathrm{Cu}_{2-x} \mathrm{~S}$ nanocrystals can be ascribed to the ability of $\mathrm{MUA}^{-}$ligands to provide both charge and steric stabilization, in contrast to $\mathrm{S}^{2-}$ ligands. The fast phase transfer observed for oleylamine-capped $\mathrm{Cu}_{2-x} \mathrm{~S}$ nanocrystals demonstrates that indeed oleylamine ligands are weakly bound to the $\mathrm{Cu}_{2-x} \mathrm{~S}$ nanocrystal surface and are thus more easily replaced than DDT ligands.

The native DDT ligands on the $\mathrm{Cu}_{2-x} \mathrm{~S}$ nanocrystals were replaced by all three ligands investigated when the ligand exchange was performed under inert atmosphere using formamide as polar solvent. However, a significant difference was observed in the kinetics of the ligand exchange for the three different replacing ligands. The kinetics of the ligand exchange was determined by the time it took for $\mathrm{Cu}_{2-x} \mathrm{~S}$ nanocrystals to transfer from the apolar to the polar phase. This phase transfer only occurs after a sufficiently large number of apolar DDT molecules has been exchanged by polar ligands. The kinetics of the phase transfer process thus reflects the cumulative rates of a series of individual ligand exchange steps. The time it takes for phase transfer to occur can then be directly related to the ligand exchange rates, being shorter for faster exchange rates. Therefore, we will discuss the kinetics of the phase transfer in terms of ligand exchange rates. The ligand exchange was fastest when using $\mathrm{S}^{2-}$. Here, phase transfer of the $\mathrm{Cu}_{2-x} \mathrm{~S}$ nanocrystals prepared by hot-injection synthesis was already observed upon stirring for $1 \mathrm{~h}$. In contrast, ligand exchange was observed only after 2 hours for MPA and only after 12 hours for MUA. The same trend in ligand exchange rates with replacing ligand was observed for nanocrystals prepared by heating-up synthesis. The origin of the difference in the ligand exchange rates with the replacing ligands is 
discussed in more detail later. Besides the dependence of the ligand exchange rate on the replacing ligand, we found that the synthesis method of the $\mathrm{Cu}_{2-x} \mathrm{~S}$ nanocrystals (hot-injection with TOPO or heating-up without TOPO) also induced differences in the ligand exchange rates. The observed trend in all experiments was that the heating-up method gives $\mathrm{Cu}_{2-x} \mathrm{~S}$ nanocrystals that are more resistant toward ligand exchange than $\mathrm{Cu}_{2-x} \mathrm{~S}$ nanocrystals prepared by hot-injection synthesis with TOPO. To better understand this difference, additional experiments were performed.

A possible factor influencing the success of ligand exchange could be the formation of superstructures by self-organization of monodisperse nanocrystals. These superstructures could hinder the ligand exchange as the nanocrystals that are packed in the interior of the superlattices are shielded from the surroundings and thus from the incoming ligands. Besides single nanocrystals (Figure 1A,D), TEM evidenced the presence of these superstructures in both the samples prepared by hot-injection synthesis (Figure 4A) and by heating-up
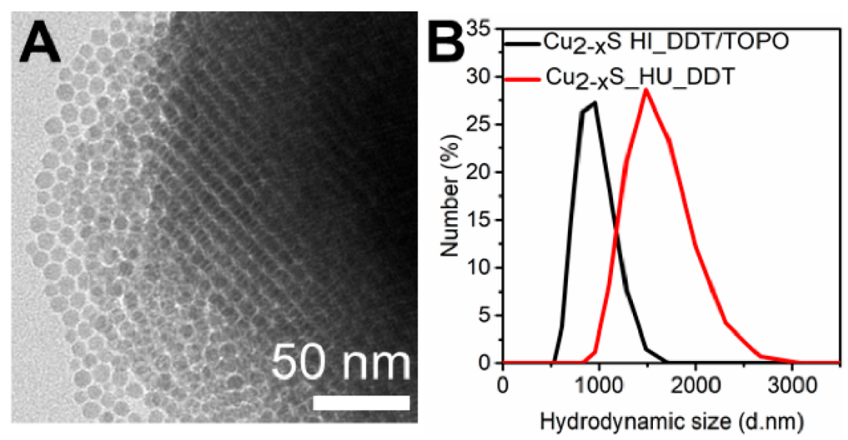

Figure 4. (A) TEM image of a superstructure formed from monodisperse $\mathrm{Cu}_{2-x} \mathrm{~S}$ nanocrystals prepared by hot-injection synthesis. (B) Hydrodynamic size of $\mathrm{Cu}_{2-x} \mathrm{~S}$ nanocrystals in toluene prepared by either hot-injection synthesis or heating-up synthesis. The large hydrodynamic size indicates the presence of nanocrystal superstructures.

synthesis. The presence of superstructures in the colloidal suspensions is verified using dynamic light scattering (DLS), where hydrodynamic sizes of $1564 \pm 316 \mathrm{~nm}$ and $932 \pm 184$ $\mathrm{nm}$ were observed for the samples prepared by heating-up and hot-injection, respectively (Figure 4b). Assuming a DDT chain length of $1.8 \mathrm{~nm},{ }^{60}$ the sizes of the $\mathrm{Cu}_{2-x} \mathrm{~S}$ nanocrystals including ligand layer are $\sim 16 \mathrm{~nm}$ for $\mathrm{HU}$ DDT/TOPO and $\sim 11 \mathrm{~nm}$ for HI DDT. For both samples, the hydrodynamic size found by DLS was much larger than the size of the $\mathrm{Cu}_{2-x} \mathrm{~S}$ nanocrystals including the ligand shell, which indicates that large nanocrystal superstructures are present in the colloidal dispersion. The larger size of the superstructures observed for the sample prepared by heating-up synthesis is attributed to the larger particle size. As can be seen from the hydrodynamic size distribution shown in Figure $4 \mathrm{~b}$, the large superstructures were predominant in both samples. Considering that superstructures were present in both samples, it is unlikely that the difference in the ligand exchange rates can be ascribed to their presence.

Another difference between the two samples is the size of the nanocrystals. Since the bond strength between ligand and nanocrystal has been reported to be size-dependent by several groups, ${ }^{61,62}$ we investigated the influence of the nanocrystal size on the rate of ligand exchange. To make nanocrystals similar in size, $\mathrm{Cu}_{2-x} \mathrm{~S}$ nanocrystals with a size of $11 \mathrm{~nm}$ were synthesized using the hot-injection method with TOPO by allowing for a longer reaction time (Figure 5a). Subsequently,
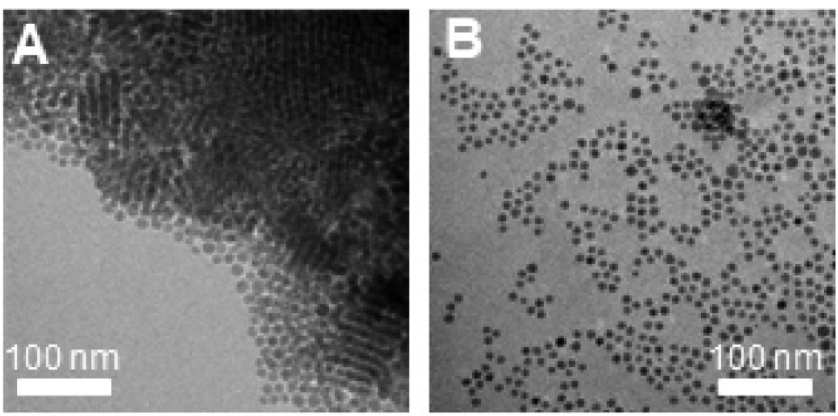

Figure 5. TEM images of $\mathrm{Cu}_{2-x} \mathrm{~S}$ nanocrystals of $\sim 11 \mathrm{~nm}$ prepared by hot-injection, (A) before and (B) after ligand exchange with MUA in water.

ligand exchange procedures using MUA in water were performed. In the ligand exchange experiments discussed above, the exchange using MUA in water occurred only for the $7 \mathrm{~nm} \mathrm{Cu} \mathrm{Cu}_{2-x} \mathrm{~S}$ nanocrystals prepared by hot-injection synthesis in the presence of TOPO, upon stirring overnight. The $12 \mathrm{~nm}$ $\mathrm{Cu}_{2-x} \mathrm{~S}$ nanocrystals prepared by heating-up synthesis did not transfer to the aqueous phase, even after stirring for 3 days. In contrast, the $11 \mathrm{~nm} \mathrm{Cu}_{2-x} \mathrm{~S}$ nanocrystals prepared by hotinjection in the presence of TOPO underwent phase transfer from the apolar to the polar phase upon stirring overnight (Figure $5 b$ ). The ligand exchange rate was thus similar for 7 and $11 \mathrm{~nm} \mathrm{Cu}_{2-x} \mathrm{~S}$ nanocrystals prepared by hot-injection synthesis. This demonstrates that the difference in the ligand exchange rates cannot be explained by nanocrystal size effects, nor by the presence of superstructures, and must thus be due to differences in the surface chemistry of the $\mathrm{Cu}_{2-x} \mathrm{~S}$ nanocrystals prepared by the two different synthesis methods.

Surface Chemistry of the $\mathrm{Cu}_{2-x} \mathrm{~S}$ Nanocrystals. As mentioned above, the nanocrystals prepared by hot-injection were synthesized in the presence of TOPO. TOPO is a widely used ligand in the synthesis of colloidal semiconductor nanocrystals, including $\mathrm{Cu}_{2-x} \mathrm{~S}$ and $\mathrm{CuInS}_{2}$, and is generally assumed to be present as a capping ligand at the nanocrystal surface after synthesis. ${ }^{34,63-65}$ Furthermore, Wang et al. reported a shape controlling effect of TOPO in the synthesis of $\mathrm{Cu}_{2-x} \mathrm{~S}$ nanocrystals, which was attributed to preferential binding of TOPO onto (001) crystal facets, thereby facilitating anisotropic growth. ${ }^{48}$ The presence of TOPO in combination with DDT at the surface of the nanocrystals synthesized by hot-injection could thus influence the ligand exchange rates. Therefore, we studied the surface species of the $\mathrm{Cu}_{2-x} \mathrm{~S}$ nanocrystals in more detail by means of X-ray photoelectron spectroscopy (XPS). Due to the limited escape depth of the generated photoelectrons, XPS is a surface sensitive technique and hence will be sensitive to probe the ligand layer around the nanocrystals and (part of) the $\mathrm{Cu}_{2-x} \mathrm{~S}$ nanocrystals as well.

XPS survey spectra of relevant samples are shown in the Supporting Information (Figure S7). From the survey scans, it can be readily observed that none of the phosphorus core lines are observed in the sample prepared by hot-injection, indicating the absence of TOPO at the nanocrystal surface (Supporting Information, Figure S8). In addition, liquid phase ${ }^{31} \mathrm{P}$ NMR was used to study the presence of TOPO bound to 
the nanocrystal surface in a sample taken directly after synthesis and of the same sample after washing with a mixture of methanol and butanol three times (see Experimental Section for details). Figure 6 a shows the ${ }^{31} \mathrm{P}$ NMR spectra of the
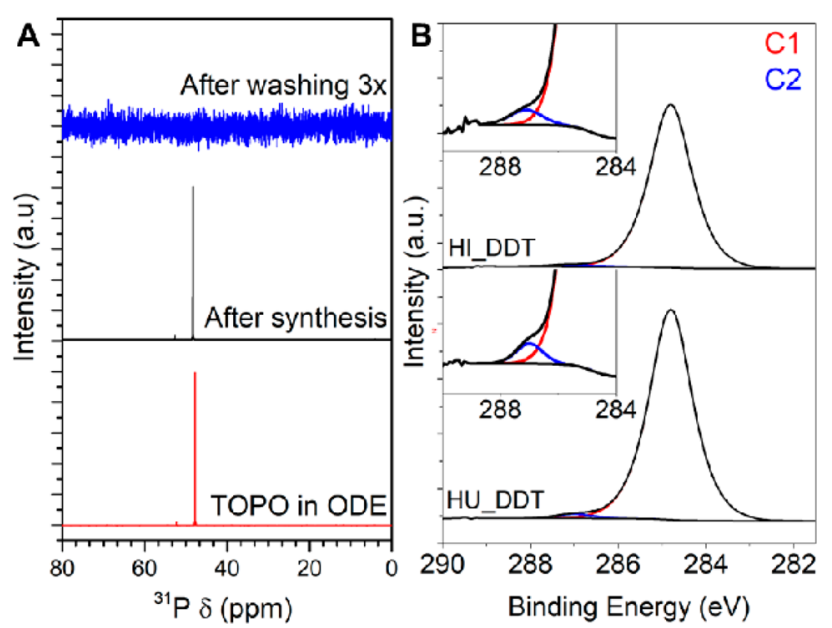

Figure 6. (A) ${ }^{31} \mathrm{P}$ NMR spectra of TOPO in ODE (reference) and of as-synthesized $\mathrm{Cu}_{2-x} \mathrm{~S}$ nanocrystals prepared by hot-injection in the presence of TOPO and after washing with a methanol/butanol mixture three times. For the washed sample, even upon zooming in no ${ }^{31} \mathrm{P}$ resonances are observed. (B) XPS spectra of the $\mathrm{C} 1 \mathrm{~s}$ region of assynthesized $\mathrm{Cu}_{2-x} \mathrm{~S}$ nanocrystals, prepared by hot-injection (top) and heating-up (bottom) synthesis protocols. The fits of the first and second carbon components ( $\mathrm{C} 1$ and $\mathrm{C} 2)$ are shown in red and blue, respectively.

unwashed sample and the sample washed three times. In the unwashed sample, a sharp resonance is present at a chemical shift of $48 \mathrm{ppm}$. By using a reference solution of TOPO in ODE, the resonance at $48 \mathrm{ppm}$ is ascribed to TOPO. Ligands bound to nanocrystal surfaces typically show broad resonances due to solvent exclusion from the ligand shell and shorter relaxation times originating from the restricted mobility of the ligands when bound to the nanocrystal surface. ${ }^{66-70}$ Hence, the sharp resonance observed for the unwashed sample indicates that after synthesis no significant amount of TOPO is strongly bound to the nanocrystal surface, but instead TOPO is present as free ligand in solution. The sample analyzed after three wash steps shows no resonances in the ${ }^{31} \mathrm{P}$ NMR spectrum, indicating that washing successfully removed free TOPO ligands from the nanocrystals dispersion and also bound TOPO from the nanocrystal surface. The absence of TOPO species at the nanocrystal surface after synthesis and washing, in combination with the finding that TOPO acts as a shape-directing ligand in $\mathrm{Cu}_{2-x} \mathrm{~S}$ nanocrystal synthesis, ${ }^{48}$ suggests that TOPO binds only weakly and dynamically to the nanocrystal surface during the synthesis and therefore does not end up at the nanocrystal surface in the equilibrium structure formed after synthesis and washing. The weak bond between TOPO and the nanocrystal surface can be rationalized in terms of Lewis acid-base interaction. ${ }^{15,24}$ TOPO binds through its oxygen lone pair to the $\mathrm{Cu}^{+}$in the $\mathrm{Cu}_{2-x} \mathrm{~S}$ nanocrystal. Since oxygen is a hard Lewis base and $\mathrm{Cu}^{+}$a soft Lewis acid, the interaction between the two species will be weak. In contrast, TOPO binds to the surface of, e.g., $\mathrm{CuInS}_{2}$ nanocrystals, ${ }^{63,64}$ which can be explained by the fact that $\mathrm{In}^{3+}$ is a strong Lewis acid and will thus bind more strongly to the strong Lewis base oxygen.

Besides TOPO, acetate was added during the hot-injection synthesis (as $\mathrm{Cu}(\mathrm{I})$ acetate) and hence could be present as a weakly binding ligand on the $\mathrm{Cu}_{2-x} \mathrm{~S}$ nanocrystals. Additionally, in the heating-up synthesis, oleic acid is used as solvent and coordinating ligand and can consequently be present in the sample. To identify the carbon species present in the assynthesized samples high-resolution XPS studies on the carbon $1 \mathrm{~s}$ core line were performed (Figure $6 \mathrm{~b}$ ). In the $\mathrm{Cu}_{2-x} \mathrm{~S}$ nanocrystals prepared by hot-injection, carbon species can originate from the thiolate ligands, 1-octadecene, or from acetate in the copper(I)acetate precursor. For the $\mathrm{Cu}_{2-x} \mathrm{~S}$ nanocrystals prepared by heating-up, carbon species can originate either from the thiolate ligands or from oleic acid
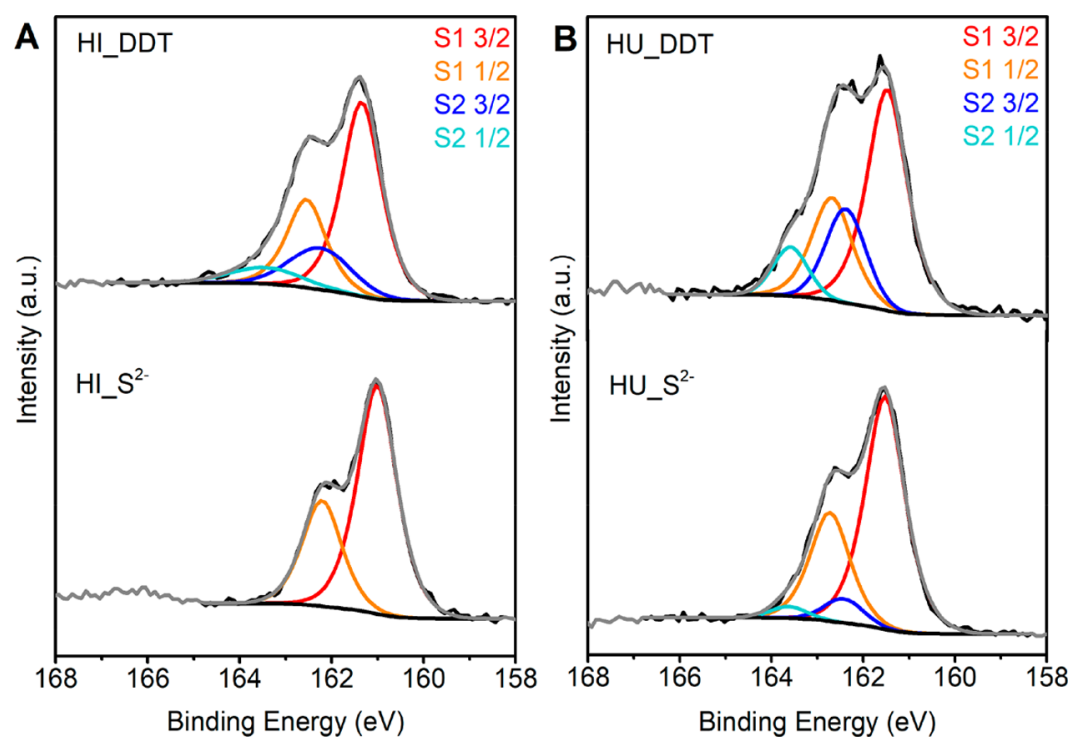

Figure 7. XPS of the sulfur $2 p$ region of $\mathrm{Cu}_{2-x} \mathrm{~S}$ nanocrystals before and after ligand exchange with $\mathrm{S}^{2-}$ prepared by (A) hot-injection and (B) heating-up synthesis protocols. The black lines indicate the experimental data. The fits of the first sulfur component are shown in red and orange, and the fit of the second sulfur component is shown in blue and light blue. 
used in the synthesis. Other solvents and antisolvents used during the synthesis and washing-up procedures have low boiling points and consequently will have evaporated in the ultrahigh vacuum applied during the XPS measurements.

For both samples, C 1s peaks are observed at a binding energy of 284.8 and $287.0 \mathrm{eV}$. The peak at a binding energy of $284.8 \mathrm{eV}$ is ascribed to carbon atoms in an aliphatic chain (CC) either originating from the thiolate ligands, 1-octadecene, oleic acid, or adventitious carbon. ${ }^{71-74}$ The peak at a binding energy of $287.0 \mathrm{eV}$ is present for both species and is ascribed to carbon bound to sulfur in thiolates. ${ }^{71}$ Both the acetate and oleic acid species will give rise to a $\mathrm{C} 1 \mathrm{~s}$ peak originating from carboxylate species $\left(\mathrm{O}=\mathrm{C}-\mathrm{O}^{-}\right)$at slightly higher binding energies in the range of $288.1-289.1 \mathrm{eV}^{71-75}$ As no peaks are present in this region in the $\mathrm{C} 1 \mathrm{~s}$ spectrum of both samples, we conclude that there was no significant amount of acetate or oleic acid present. Moreover, these results show that both types of as-synthesized nanocrystals were capped solely by thiol ligands.

To further investigate the surface of the $\mathrm{Cu}_{2-x} \mathrm{~S}$ nanocrystals, high-resolution XPS studies on the sulfur $2 p$ core line were performed (Figure 7). The as-synthesized nanocrystals capped with the thiol species were compared to the $S 2 p$ regions of the nanocrystals after ligand exchange with $\mathrm{S}^{2-}$. For a single sulfur component, a set of spin-orbit coupled peaks $\left(2 \mathrm{p}_{3 / 2}\right.$ and $\left.2 p_{1 / 2}\right)$ is expected with an intensity ratio of $1: 2$ and a spinorbit coupling split of $\sim 1.2 \mathrm{eV}$. The $\mathrm{S} 2 \mathrm{p}$ regions of the assynthesized samples clearly exhibited a second component and were therefore fitted using two sets of spin-orbit coupled peaks. In agreement with recent studies, ${ }^{40,71}$ we ascribe the component at an S $2 \mathrm{p}_{3 / 2}$ binding energy of $161.5 \mathrm{eV}$ to sulfur in the $\mathrm{Cu}_{2-x} \mathrm{~S}$ nanocrystal lattice and possibly crystal-bound thiolate ligands. In the crystal-bound thiolate ligands, sulfur atoms are bound into higher coordination sites within the crystal lattice and hence the binding energy is very similar to the sulfides in the $\mathrm{Cu}_{2-x} \mathrm{~S}$ nanocrystal. ${ }^{40}$ The second component has a higher $\mathrm{S} 2 \mathrm{p}_{3 / 2}$ binding energy of $162.4 \mathrm{eV}$ and hence is ascribed to a sulfur species different from the sulfur in the $\mathrm{Cu}_{2-x} \mathrm{~S}$ lattice. The identical binding energy of $162.4 \mathrm{eV}$ observed for the second sulfur component in the samples prepared by different synthesis methods suggests that the second sulfur component originates from the same sulfur species in both samples. This second S 2 p component can be ascribed to surface-bound thiolate (i.e., thiolate bound to surface sites with lower coordination numbers), as described by Turo et al. ${ }^{40}$ Other sulfur species possibly associated with this high binding energy $S 2 p$ component are thioethers forming a ligand double layer, similar to that recently described by Gromova et al. for $\mathrm{CuInS}_{2}$ nanocrystals prepared by a heating-up synthesis in the presence of DDT. ${ }^{46}$

For the $\mathrm{Cu}_{2-x} \mathrm{~S}$ nanocrystals prepared by hot-injection in the presence of TOPO, the second sulfur component holds $9 \%$ of the total sulfur species, whereas this is $28 \%$ for the nanocrystals prepared by heating-up synthesis in the absence of TOPO. This suggests a denser layer of surface-bound thiolate species or thioether species around the nanocrystals prepared by the heating-up synthesis. Figure 7 (bottom) shows the highresolution XPS spectra in the $S 2 p$ region of the $\mathrm{Cu}_{2-x} S$ nanocrystals after ligand exchange with $\mathrm{S}^{2-}$. For the nanocrystals prepared by hot-injection, the $S 2 p$ feature is best fitted with a single set of spin-orbit coupled peaks. Upon ligand exchange with $S^{2-}$, the second component originating from the ligand layer is thus completely removed. In contrast, the second set of spin-orbit coupled peaks is still clearly present for the nanocrystals prepared by heating-up, indicating only partial removal of the surface-bound alkanethiols. The trend of ligand removal found with XPS studies correlates with the success and kinetics of the ligand exchange procedures discussed above, where it was found that DDT molecules on $\mathrm{Cu}_{2-x} \mathrm{~S}$ nanocrystals prepared by hot-injection in the presence of TOPO were more easily exchanges by the replacing ligands.

Model for Ligand Exchange on DDT-Capped $\mathrm{Cu}_{2-x} \mathrm{~S}$ Nanocrystals. Based on the observations above we propose a model for the ligand exchange that explains the observed differences in the exchange rates. In the ligand exchange protocol developed in our work a two layer system is formed in which the apolar layer contains the $\mathrm{Cu}_{2-x} \mathrm{~S}$ nanocrystals capped by the native thiolate ligands and the polar formamide layer contains the replacing negatively charged ligands $\left(\mathrm{MPA}^{-}\right.$, $\mathrm{MUA}^{-}$, and $\mathrm{S}^{2-}$ ) and, later in the process, also the negatively charged $\mathrm{Cu}_{2-x} \mathrm{~S}$ nanocrystals capped with the replacing ligands. As the two solvents are immiscible and the replacing ligands and the charged nanocrystals do not disperse in the apolar phase, while the nanocrystals with the native DDT ligands do not disperse in the polar phase, the ligand exchange must necessarily take place at the interface between the polar and the apolar layers. The native DDT ligand is bound to the nanocrystal surface through its sulfur atom that donates an unshared electron pair and coordinates to the $\mathrm{Cu}^{+}{ }^{+15}$ In the ligand exchange reactions, the negatively charged replacing ligand $\left(\mathrm{MPA}^{-}, \mathrm{MUA}^{-}\right.$, and $\left.\mathrm{S}^{2-}\right)$ will coordinate its electronrich sulfur species to the $\mathrm{Cu}^{+}$site on the nanocrystal, ${ }^{24}$ thereby weakening the bond between the $\mathrm{Cu}^{+}$and the donor sulfur atom of the DDT ligands, which will eventually force the native thiolate ligand to leave the nanocrystal surface. When part of the native ligands are exchanged for charged species, the nanocrystals will be trapped at the interface since they will become charged (Figure 8). If most native ligands are

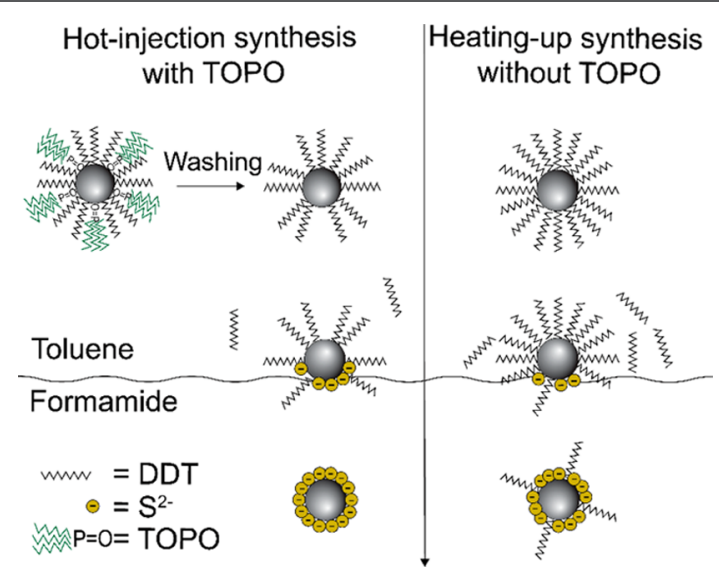

Figure 8. Schematic illustration of a ligand exchange of DDT for $\mathrm{S}^{2-}$ and subsequent phase transfer from apolar toluene to polar formamide. The $\mathrm{Cu}_{2-x} \mathrm{~S}$ nanocrystals prepared by hot-injection synthesis have a less dense ligand layer than the nanocrystals prepared by heating-up synthesis due to the removal of TOPO from the surface of the nanocrystal after synthesis and washing. Consequently, the nanocrystal surface is more accessible to the incoming ligands, resulting in a faster ligand exchange. Upon ligand exchange on nanocrystals prepared by hot-injection synthesis, all thiolate ligands are removed from the nanocrystal surface. In contrast, upon ligand exchange on nanocrystals prepared by heating-up synthesis, some thiolate ligands remain on the surface. 
exchanged for charged replacing ligands, thereby making the nanocrystal sufficiently negatively charged, the nanocrystal will transfer to the polar solvent (Figure 8). As described above, we found that the polarity of the solvent influences the success of ligand exchange. This can be understood by the good ability of the highly polar formamide to shield the charged intermediates. Similarly, the higher stability of the nanocrystals in solvents with higher polarity (e.g., formamide) results from the better shielding of the charged nanocrystals.

The observed dependence of the ligand exchange rate on the incoming ligand (viz., highest for $\mathrm{S}^{2-}$, followed by $\mathrm{MPA}^{-}$and then $\mathrm{MUA}^{-}$) can be rationalized in terms of the accessibility of the nanocrystal surface due to variable degrees of steric hindrance. The hydrophobic ligand layer at the nanocrystal surface acts as a barrier around the nanocrystal. The small $\mathrm{S}^{2-}$ ions can more easily diffuse through this hydrophobic layer and reach the surface $\mathrm{Cu}^{+}$sites, whereas the bulky MUA molecule cannot easily access the surface sites, which results in much slower ligand exchange rates. The observed difference in the ligand exchange rates for the $\mathrm{Cu}_{2-x} \mathrm{~S}$ nanocrystals prepared by hot-injection or heating-up can be explained by the ligand layer at the surface of the nanocrystals. Although we cannot identify the exact nature of the ligand layer, the XPS studies discussed above indicate a denser layer of thiolate ligands around $\mathrm{Cu}_{2-x} \mathrm{~S}$ nanocrystals prepared by the heating-up synthesis, i.e., in the absence of TOPO. The fact that the native ligand layer is less dense for the nanocrystals prepared through the hot-injection method can be understood from the synthesis conditions used. In the hot-injection synthesis, TOPO is used as a coordinating ligand. As has been shown by Wang et al. TOPO has a shape directing effect in the synthesis of $\mathrm{Cu}_{2-x} \mathrm{~S}$ nanocrystals, implying that it dynamically binds to the nanocrystals surface during its growth. ${ }^{48}$ The bulky nature of TOPO will thus prevent the formation of a dense layer of thiolates around the growing $\mathrm{Cu}_{2-x} \mathrm{~S}$ nanocrystals. As shown by the XPS and ${ }^{31} \mathrm{P}$ NMR studies discussed above, TOPO is no longer present on the $\mathrm{Cu}_{2-x} \mathrm{~S}$ nanocrystals after the synthesis, implying that it has been removed from the surface, thereby leaving vacant sites on the nanocrystal surface. Consequently, the $\mathrm{Cu}_{2-x} \mathrm{~S}$ nanocrystals prepared by hotinjection synthesis in the presence of TOPO have a less dense layer of thiolate ligands at the nanocrystal surface than nanocrystals prepared by the heating-up synthesis. The denser, hydrophobic native ligand layer at the surface of the nanocrystals prepared by the heating-up method will make the surface sites less accessible for the charged incoming ligands and hence will significantly slow down the ligand exchange kinetics.

Additionally, $\mathrm{Cu}_{2-x} \mathrm{~S}$ nanocrystals prepared by hot-injection show a single sulfur species upon ligand exchange with $\mathrm{S}^{2-}$, indicating that all surface-bound thiolate ligands are removed from the nanocrystal surface upon ligand exchange. In contrast, nanocrystals prepared using heating-up synthesis show residual surface-bound thiolate species at their surface upon ligand exchange (see Figure 7 and XPS discussion above). Consequently, the nanocrystals are likely capped with a combination of remaining thiolate species and sulfides, as is schematically represented in Figure 8. However, since the nanocrystals do show colloidal stability in formamide, the nanocrystal surface is covered by sufficient negatively charged sulfide anions to stabilize the nanocrystals in formamide.

\section{CONCLUSIONS}

In this paper we present effective ligand exchange procedures of DDT on $\mathrm{Cu}_{2-x} \mathrm{~S}$ nanocrystals for $\mathrm{MPA}^{-}, \mathrm{MUA}^{-}$, and $\mathrm{S}^{2-}$ from $\mathrm{Na}_{2} \mathrm{~S}$ in formamide under inert atmosphere. The ligand exchange procedures did not significantly affect the size, shape, and optical properties of the nanocrystals. The resulting hydrophilic $\mathrm{Cu}_{2-x} \mathrm{~S}$ nanocrystals have excellent colloidal stability in formamide. Water-dispersible $\mathrm{Cu}_{2-x} \mathrm{~S}$ nanocrystals can be easily obtained by precipitation of the nanocrystals, followed by redispersion in water. In addition, the ligand exchange rates for DDT-capped $\mathrm{Cu}_{2-x} \mathrm{~S}$ nanocrystals depended on the preparation method, being much slower for $\mathrm{Cu}_{2-x} \mathrm{~S}$ nanocrystals prepared by heating-up than by hot-injection synthesis. XPS studies revealed that the differences in the ligand exchange rates can be attributed to the surface chemistry of the $\mathrm{Cu}_{2-x} \mathrm{~S}$ nanocrystals, where nanocrystals prepared by heating-up synthesis had a denser ligand layer in comparison to nanocrystals prepared by hot-injection in the presence of TOPO. The less dense native thiolate ligand layer on the surface of the nanocrystals prepared by hot-injection originates from the presence of TOPO during the synthesis, which prevents the formation of a dense thiolate layer around the nanocrystals during their growth, thus leaving vacant surface sites after being removed upon washing after the synthesis. The dense native thiolate ligand layer makes the nanocrystal surface less accessible for the charged incoming ligands, hence significantly slowing down the ligand exchange rates. The facile ligand exchange procedures developed in our work open up opportunities for the use of high-quality colloidal $\mathrm{Cu}_{2-x} \mathrm{~S}$ nanocrystals prepared with thiols in various applications, such as photo- and electrocatalysis, photothermal therapy, or solution-processable devices (flexible conductive films, sensors, solar cells).

\section{EXPERIMENTAL SECTION}

Materials. Copper(II) sulfate pentahydrate $\left(\mathrm{CuSO}_{4} \cdot 5 \mathrm{H}_{2} \mathrm{O}\right.$ 99.999\%), copper(I) acetate (CuAc, 97\%), copper(I) chloride (CuCl, 99.995\%), 1-dodecanethiol (DDT, $\geq 98 \%)$, oleic acid (OA, 90\%), 1-octadecene (ODE, 90\%), trioctylphosphine oxide (TOPO, $99 \%$ ), oleylamine (OLAM, 70\%), sodium sulfide $\left(\mathrm{Na}_{2} \mathrm{~S}\right), 11$ mercaptoundecanoic acid (MUA, 98\%), 3-mercaptopropionic acid (99\%), tetramethylammonium hydroxide pentahydrate (TMAH, $\geq 97 \%$ ), formamide (FA, 98\%), chloroform, anhydrous toluene, methanol, butanol, ethanol, hexane, and acetonitrile were purchased from Sigma-Aldrich. ODE and TOPO were degassed at $120^{\circ} \mathrm{C}$ for 3 $\mathrm{h}$ prior to use. Formamide was degassed at $120^{\circ} \mathrm{C}$ for $2 \mathrm{~h}$ prior to use. All other reagents were used as received.

Synthesis of Colloidal DDT-Capped $\mathrm{Cu}_{2-x} \mathrm{~S}$ Nanocrystals by the Heating-Up Method. $\mathrm{Cu}_{2-x} \mathrm{~S}$ nanocrystals of $12 \mathrm{~nm}$ diameter were synthesized according to the method described by van der Stam et al. ${ }^{49} \mathrm{~A}$ total of $205 \mathrm{mg}(0.8 \mathrm{mmol})$ of $\mathrm{CuSO}_{4} \cdot 5 \mathrm{H}_{2} \mathrm{O}, 7.5 \mathrm{~mL}$ of $\mathrm{DDT}$, and $6 \mathrm{~mL}$ of $\mathrm{OA}$ were mixed in a round-bottom flask. The mixture was gradually heated to $200{ }^{\circ} \mathrm{C}$ under nitrogen protection in a Schlenk line. Subsequently, the solution was kept at $200{ }^{\circ} \mathrm{C}$ for $2 \mathrm{~h}$. After that, the reaction mixture was washed three times by addition of an excess of methanol and butanol (1:1 ratio), followed by centrifugation at $2500 \mathrm{rpm}$ for $5 \mathrm{~min}$. The nanocrystals were redispersed in $10 \mathrm{~mL}$ of anhydrous toluene.

Synthesis of Colloidal $\mathrm{Cu}_{2-x} \mathrm{~S}$ Nanocrystals by the HotInjection Method. $\mathrm{Cu}_{2-x} \mathrm{~S}$ nanocrystals of various sizes were synthesized by adaptation of the method described by Xia et al. ${ }^{34}$ In a round-bottom flask, $0.13 \mathrm{~g}(1 \mathrm{mmol})$ of $\mathrm{Cu}(\mathrm{I}) \mathrm{Ac}$ and $3.6 \mathrm{~g}(9.3$ mmol) of TOPO were added to $20 \mathrm{~mL}$ of ODE. First, the mixture was degassed at $100{ }^{\circ} \mathrm{C}$ for $1 \mathrm{~h}$. Subsequently, the flask was purged with nitrogen, and subsequent steps of the synthesis were performed under nitrogen flow in a Schlenk line. The reaction mixture was 
heated to $210^{\circ} \mathrm{C}$. At $160^{\circ} \mathrm{C}, 5 \mathrm{~mL}$ of DDT was quickly injected into the flask. The nanocrystals were allowed to grow for $1 \mathrm{~h}$ at $210{ }^{\circ} \mathrm{C}$. After reaction, the nanocrystals were washed using the method described above and redispersed in $10 \mathrm{~mL}$ of anhydrous toluene.

Synthesis of Colloidal OLAM-Capped $\mathrm{Cu}_{2-x} \mathrm{~S}$ Nanocrystals by the Heating-Up Method. OLAM-capped $\mathrm{Cu}_{2-x} \mathrm{~S}$ nanocrystals of $7 \mathrm{~nm}$ diameter were synthesized in a Schlenk line under nitrogen according to a method described by Williamson et al. ${ }^{76}$ A S-OLAM precursor solution was prepared by dissolving $1.6 \mathrm{~g}(50 \mathrm{mmol})$ of $\mathrm{S}$ in $7 \mathrm{~mL}$ of OLAM and $3 \mathrm{~mL}$ of ODE at $110^{\circ} \mathrm{C}$. The copper precursor solution was prepared by dissolving $6 \mathrm{~g}(60 \mathrm{mmol})$ of $\mathrm{CuCl}$ in $42 \mathrm{~mL}$ of OLAM and $18 \mathrm{~mL}$ of ODE in a round-bottom flask at $110^{\circ} \mathrm{C}$. Both precursor solutions were cooled to $50{ }^{\circ} \mathrm{C}$, after which $6 \mathrm{~mL}$ of the S-OLAM precursor was added to the $\mathrm{Cu}$-precursor solution. The mixture was left stirring at $50{ }^{\circ} \mathrm{C}$ for $5 \mathrm{~min}$. Subsequently, the temperature was increased to $185{ }^{\circ} \mathrm{C}$ in $30 \mathrm{~min}$ and maintained for 2 h. After that the reaction mixture was cooled using a water bath and addition of $60 \mathrm{~mL}$ of hexane, which also decreased the viscosity of the mixture. The product was purified by washing with ethanol, followed by centrifugation at $2500 \mathrm{rpm}$ for $5 \mathrm{~min}$. The $\mathrm{Cu}_{2-x} \mathrm{~S}$ nanocrystals were redispersed in $75 \mathrm{~mL}$ of $n$-hexane.

Ligand Exchange Using $\mathrm{Na}_{2} \mathrm{~S}$. The phase transfer procedure using $\mathrm{Na}_{2} \mathrm{~S}$ was done using an adaptation of a method described by Nag et al. ${ }^{24}$ For a typical ligand exchange, $1 \mathrm{~mL}$ of $\mathrm{Na}_{2} \mathrm{~S}$ in formamide or demineralized water $(5 \mathrm{mg} / \mathrm{mL})$ was mixed with $1 \mathrm{~mL}$ of $\mathrm{Cu}_{2-x} \mathrm{~S}$ nanocrystals in toluene $(\sim 5 \mathrm{mg} / \mathrm{mL})$. The experiments using formamide as the polar solvent were also performed under inert atmosphere in a nitrogen glovebox $\left(\leq 6 \mathrm{ppm}\right.$ of $\left.\mathrm{O}_{2}\right)$. A biphase was formed, with the colorless $\mathrm{Na}_{2} \mathrm{~S}$ solution on the bottom and the black $\mathrm{Cu}_{2-x} \mathrm{~S}$ nanocrystals suspension in toluene on top (Supporting Information, Figure S1). Upon ligand exchange of the native apolar ligands to sulfide, the black $\mathrm{Cu}_{2-x} \mathrm{~S}$ nanocrystals transferred to the polar formamide layer. Subsequently, the apolar layer was removed and the remaining polar phase containing the $\mathrm{Cu}_{2-x} \mathrm{~S}$ nanocrystals was washed with toluene to remove the remaining apolar species. The $\mathrm{Cu}_{2-x} \mathrm{~S}$ nanocrystals were precipitated by adding an excess of acetonitrile, followed by centrifugation for $2 \mathrm{~min}$ at $2000 \mathrm{rpm}$. The purified sulfide-capped $\mathrm{Cu}_{2-x} \mathrm{~S}$ nanocrystals were redispersed in $2 \mathrm{~mL}$ of degassed formamide and stored under nitrogen atmosphere.

Ligand Exchange Using MUA or MPA. The phase transfer procedure using MUA or MPA was based on a method described by Xia et al. ${ }^{34}$ A total of $1.4 \mathrm{mmol}$ of MUA or MPA was dissolved in 30 $\mathrm{mL}$ of demineralized $\mathrm{H}_{2} \mathrm{O}$ or formamide. In the case of MUA, TMAH was added to increase the $\mathrm{pH}$ to 11 and deprotonate the MUA. In a typical ligand exchange, $4 \mathrm{~mL}$ of a $\mathrm{Cu}_{2-x} \mathrm{~S}$ nanocrystal suspension in toluene $(\sim 0.75 \mathrm{mg} / \mathrm{L})$ was mixed with $5.8 \mathrm{~mL}$ of the MUA or MPA solutions. A two-layer system was formed, with the $\mathrm{Cu}_{2-x} \mathrm{~S}$ nanocrystals in toluene on the bottom and the polar MUA or MPA solution on top. The experiments using formamide as the polar solvent were also performed under inert atmosphere in a nitrogen glovebox $\left(\leq 6 \mathrm{ppm}\right.$ of $\left.\mathrm{O}_{2}\right)$. The ligand exchange was successful when upon stirring the black $\mathrm{Cu}_{2-x} \mathrm{~S}$ nanocrystals transferred to the polar phase. After centrifugation for $1 \mathrm{~min}$ at $1000 \mathrm{rpm}$, the polar layer containing the $\mathrm{Cu}_{2-x} \mathrm{~S}$ nanocrystals was collected and washed by adding excess ethanol, followed by centrifugation for $5 \mathrm{~min}$ at 3000 rpm. The purified MUA- or MPA-capped $\mathrm{Cu}_{2-x} \mathrm{~S}$ nanocrystals were redispersed in demineralized water or formamide.

Transmission Electron Microscopy (TEM). Samples for TEM analysis were prepared by drop-casting the $\mathrm{Cu}_{2-x} \mathrm{~S}$ nanocrystal suspension on a carbon-coated 200 mesh copper TEM grid. TEM analysis was performed using a FEI Tecnai- 12 microscope operating at $120 \mathrm{kV}$ or a FEI Technai-20 microscope operating at $200 \mathrm{kV}$.

$\mathrm{X}$-ray Diffraction (XRD). Samples for XRD analysis were prepared by precipitating the $\mathrm{Cu}_{2-x} \mathrm{~S}$ nanocrystals with an antisolvent and subsequently drying the obtained powder under vacuum. The dried $\mathrm{Cu}_{2-x} \mathrm{~S}$ nanocrystal powder was spread over a $\mathrm{Si}$ wafer. XRD measurements were performed with a Bruker D2 Phaser, equipped with a Co K $\alpha$ X-ray source with a wavelength of $1.79026 \AA$.

$\zeta$-Potential and Dynamic Light Scattering (DLS). $\zeta$-Potential and DLS measurements were performed on a Zetasizer Nano ZS from
Malvern Instruments using a dip cell with palladium electrodes with 2 mm spacing in a PCS1115 cuvette and a measurement angle of $173^{\circ}$. Measurements were corrected by the instrument software for solvent (formamide or toluene), refractive index, temperature, and viscosity. Measurements were done in the automatic mode and repeated five times for each sample in order to obtain reliable results.

X-ray Photoelectron Spectroscopy (XPS). Sample preparation for XPS was done under air-free conditions to prevent oxidation and chemisorption of $\mathrm{CO}_{2}$ on the samples. Samples were mounted in a glovebox and transferred into the spectrometer in a vacuum transfer module. The as-synthesized samples with the original apolar ligands were drop casted on a clean tantalum sample holder in order to avoid the influence of carbon tape. Samples after ligand exchange to $\mathrm{S}^{2-}$ were dried under vacuum and stuck onto the tantalum sample holder as powder using carbon tape. XPS spectra were taken with a Thermo Scientific K-Alpha spectrometer using a $72 \mathrm{~W}$ monochromated $\mathrm{Al} \mathrm{K \alpha}$ source $(h \nu=1486.6 \mathrm{eV})$. The X-rays are microfocused at the source to give a spot size on the sample of $400 \mu \mathrm{m}$ in diameter. The analyzer is a double focusing $180^{\circ}$ hemisphere with mean radius of $125 \mathrm{~mm}$, run in constant analyzer energy (CAE) mode. The pass energy was set to $200 \mathrm{eV}$ for survey scans and $50 \mathrm{eV}$ for high resolution regions. Data analysis was done using CasaXPS software. Binding energies were calibrated by setting the lowest energy C 1 s peak to $284.8 \mathrm{eV}$.

${ }^{31} \mathrm{P}$ NMR Spectroscopy. ${ }^{31} \mathrm{P}$ NMR measurements were done on a reference solution of TOPO in ODE, the $\mathrm{Cu}_{2-x} \mathrm{~S}$ nanocrystal reaction mixture directly after synthesis and after washing three times. The reaction mixture and reference solution were both diluted with $\mathrm{CDCl}_{3}$ to a concentration half of the original in order to perform a lock procedure. The washed sample was dried under vacuum overnight after the third wash step and subsequently redispersed in $\mathrm{CDCl}_{3}$. Measurements were performed on an Aligent MRF400 spectrometer at $25{ }^{\circ} \mathrm{C}$ and $161 \mathrm{MHz}$.

\section{ASSOCIATED CONTENT}

\section{Supporting Information}

The Supporting Information is available free of charge on the ACS Publications website at DOI: 10.1021/acs.chemmater.8b04614.

Zeta potential measurements for $\mathrm{Cu}_{2-x} \mathrm{~S}$ nanocrystals after ligand exchange. Additional TEM images and XRD patterns of $\mathrm{Cu}_{2-x} \mathrm{~S}$ nanocrystals after ligand exchange. XPS survey scans for relevant samples (PDF)

\section{AUTHOR INFORMATION}

\section{Corresponding Author}

*(Celso de Mello Donega) E-mail: c.demello-donega@uu.nl. ORCID $\odot$

Christina H. M. van Oversteeg: 0000-0002-5634-684X

Jan P. Hofmann: 0000-0002-5765-1096

Emiel J. M. Hensen: 0000-0002-9754-2417

Petra E. de Jongh: 0000-0002-2216-2620

Celso de Mello Donega: 0000-0002-4403-3627

\section{Notes}

The authors declare no competing financial interest.

\section{ACKNOWLEDGMENTS}

This work was supported by The Netherlands Center for Multiscale Catalytic Energy Conversion (MCEC), an NWO Gravitation program funded by the Ministry of Education, Culture and Science of the government of The Netherlands. P.E.d.J. acknowledges funding from the European Research Council (ERC) under the European Union's Horizon 2020 research and innovation programme (ERC-2014-CoG No. 648991). The authors are grateful to Johann Jastrzebski 
(Utrecht University) for his assistance with ${ }^{31} \mathrm{P}$ NMR measurements.

\section{REFERENCES}

(1) Goel, S.; Chen, F.; Ehlerding, E. B.; Cai, W. Intrinsically Radiolabeled Nanoparticles: An Emerging Paradigm. Small 2014, 10, $3825-3830$.

(2) Coughlan, C.; Ibáñez, M.; Dobrozhan, O.; Singh, A.; Cabot, A.; Ryan, K. M. Compound Copper Chalcogenide Nanocrystals. Chem. Rev. 2017, 117, 5865-6109.

(3) Goel, S.; Chen, F.; Cai, W. Synthesis and Biomedical Applications of Copper Sulfide Nanoparticles: From Sensors to Theranostics. Small 2014, 10, 631-645.

(4) Wang, S.; Riedinger, A.; Li, H.; Fu, C.; Liu, H.; Li, L.; Liu, T.; Tan, L.; Barthel, M. J.; Pugliese, G.; et al. Plasmonic Copper Sulfide Nanocrystals Exhibiting Near-Infrared Photothermal and Photodynamic Therapeutic Effects. ACS Nano 2015, 9, 1788-1800.

(5) Zhao, Y.; Burda, C. Development of Plasmonic Semiconductor Nanomaterials with Copper Chalcogenides for a Future with Sustainable Energy Materials. Energy Environ. Sci. 2012, 5, 55645576.

(6) Luther, J. M.; Jain, P. K.; Ewers, T.; Alivisatos, A. P. Localized Surface Plasmon Resonances Arising from Free Carriers in Doped Quantum Dots. Nat. Mater. 2011, 10, 361-366.

(7) Abdelhady, A. L.; Ramasamy, K.; Malik, M. A.; O’Brien, P.; Haigh, S. J.; Raftery, J. New Routes to Copper Sulfide Nanostructures and Thin Films. J. Mater. Chem. 2011, 21, 17888-17895.

(8) Van Der Stam, W.; Gudjonsdottir, S.; Evers, W. H.; Houtepen, A. J. Switching between Plasmonic and Fluorescent Copper Sulfide Nanocrystals. J. Am. Chem. Soc. 2017, 139, 13208-13217.

(9) Shao, P.; Ci, S.; Yi, L.; Cai, P.; Huang, P.; Cao, C.; Wen, Z. Hollow $\mathrm{CuS}$ Microcube Electrocatalysts for $\mathrm{CO}_{2}$ Reduction Reaction. ChemElectroChem 2017, 4, 2593-2598.

(10) Zhao, Z.; Peng, X.; Liu, X.; Sun, X.; Shi, J.; Han, L.; Li, G.; Luo, J. Efficient and Stable Electroreduction of $\mathrm{CO}_{2}$ to $\mathrm{CH}_{4}$ on $\mathrm{CuS}$ Nanosheet Arrays. J. Mater. Chem. A 2017, 5, 20239-20243.

(11) Van Der Stam, W.; Berends, A. C.; De Mello Donega, C. Prospects of Colloidal Copper Chalcogenide Nanocrystals. ChemPhysChem 2016, 17, 559-581.

(12) Zhuang, Z.; Peng, Q.; Zhang, B.; Li, Y. Controllable Synthesis of $\mathrm{Cu}_{2} \mathrm{~S}$ Nanocrystals and Their Assembly into a Superlattice. J. Am. Chem. Soc. 2008, 130, 10482-10483.

(13) Zhao, Y.; Pan, H.; Lou, Y.; Qiu, X.; Zhu, J.; Burda, C. Plasmonic $\mathrm{Cu}_{2-\mathrm{x}} \mathrm{S}$ Nanocrystals: Optical and Structural Properties of Copper-Deficient Copper(I) Sulfides. J. Am. Chem. Soc. 2009, 131, 4253-4261.

(14) Wadia, C.; Alivisatos, A. P.; Kammen, D. M. Materials Availability Expands the Opportunity for Large-Scale Photovoltaics Deployment. Environ. Sci. Technol. 2009, 43, 2072-2077.

(15) Donegá, C. de M Synthesis and Properties of Colloidal Heteronanocrystals. Chem. Soc. Rev. 2011, 40, 1512-1546.

(16) Li, X.; Shen, H.; Niu, J.; Li, S.; Zhang, Y.; Wang, H.; Li, L. S. Columnar Self-Assembly of $\mathrm{Cu}_{2} \mathrm{~S}$ Hexagonal Nanoplates Induced by Tin(IV)-X Complex as Inorganic Surface Ligand. J. Am. Chem. Soc. 2010, 132, 12778-12779.

(17) Sigman, M. B.; Ghezelbash, A.; Hanrath, T.; Saunders, A. E.; Lee, F.; Korgel, B. A. Solventless Synthesis of Monodisperse $\mathrm{Cu}_{2} \mathrm{~S}$ Nanorods, Nanodisks, and Nanoplatelets. J. Am. Chem. Soc. 2003, $125,16050-16057$.

(18) Li, X.; Wang, M.; Shen, H.; Zhang, Y.; Wang, H.; Li, L. S. Inorganic Sn-X-Complex-Induced 1D, 2D, and 3D Copper Sulfide Superstructures from Anisotropic Hexagonal Nanoplate Building Blocks. Chem. - Eur. J. 2011, 17, 10357-10364.

(19) Li, W.; Shavel, A.; Guzman, R.; Rubio-Garcia, J.; Flox, C.; Fan, J.; Cadavid, D.; Ibáñez, M.; Arbiol, J.; Morante, J. R.; et al. Morphology Evolution of $\mathrm{Cu}_{2-\mathrm{X}} \mathrm{S}$ Nanoparticles: From Spheres to Dodecahedrons. Chem. Commun. (Cambridge, U. K.) 2011, 47, 10332-10334.
(20) Tang, A.; Qu, S.; Li, K.; Hou, Y.; Teng, F.; Cao, J.; Wang, Y.; Wang, Z. One-Pot Synthesis and Self-Assembly of Colloidal Copper(I) Sulfide Nanocrystals. Nanotechnology 2010, 21, 285602.

(21) Van Der Stam, W.; Akkerman, Q. A.; Ke, X.; Van Huis, M. A.; Bals, S.; de Mello Donega, C. Solution-Processable Ultrathin Size- and Shape-Controlled Colloidal $\mathrm{Cu}_{2-\mathrm{x}} \mathrm{S}$ Nanosheets. Chem. Mater. 2015, 27, 283-291.

(22) van der Stam, W.; Gradmann, S.; Altantzis, T.; Ke, X.; Baldus, M.; Bals, S.; de Mello Donega, C. Shape Control of Colloidal $\mathrm{Cu}_{2-x} S$ Polyhedral Nanocrystals by Tuning the Nucleation Rates. Chem. Mater. 2016, 28, 6705-6715.

(23) Van Embden, J.; Chesman, A. S. R.; Jasieniak, J. J. The Heat-Up Synthesis of Colloidal Nanocrystals. Chem. Mater. 2015, 27, 22462285.

(24) Nag, A.; Kovalenko, M. V.; Lee, J. S.; Liu, W.; Spokoyny, B.; Talapin, D. V. Metal-Free Inorganic Ligands for Colloidal Nanocrystals: $\mathrm{S}^{2-}, \mathrm{HS}^{-}, \mathrm{Se}^{2-}, \mathrm{HSe}^{-}, \mathrm{Te}^{2-}, \mathrm{HTe}^{-}, \mathrm{TeS}_{3}{ }^{2-}, \mathrm{OH}^{-}$, and $\mathrm{NH}_{2}{ }^{-}$as Surface Ligands. J. Am. Chem. Soc. 2011, 133, 10612-10620.

(25) Kovalenko, M. V.; Scheele, M.; Talapin, D. V. Colloidal Nanocrystals with Molecular Metal Chalcogenide Surface Ligands. Science 2009, 324, 1417-1420.

(26) Kovalenko, M. V.; Bodnarchuk, M. I.; Zaumseil, J.; Lee, J. S.; Talapin, D. V. Expanding the Chemical Versatility of Colloidal Nanocrystals Capped with Molecular Metal Chalcogenide Ligands. J. Am. Chem. Soc. 2010, 132, 10085-10092.

(27) Gugula, K.; Stegemann, L.; Cywiński, P. J.; Strassert, C. A.; Bredol, M. Facile Surface Engineering of $\mathrm{CuInS}_{2} / \mathrm{ZnS}$ Quantum Dots for LED down-Converters. RSC Adv. 2016, 6, 10086-10093.

(28) Wuister, S. F.; De Mello Donegá, C.; Meijerink, A. Luminescence Temperature Antiquenching of Water-Soluble CdTe Quantum Dots: Role of the Solvent. J. Am. Chem. Soc. 2004, 126, 10397-10402.

(29) Bagaria, H. G.; Ada, E. T.; Shamsuzzoha, M.; Nikles, D. E.; Johnson, D. T. Understanding Mercapto Ligand Exchange on the Surface of FePt Nanoparticles. Langmuir 2006, 22, 7732-7737.

(30) Chan, W. C. W.; Nie, S. Quantum Dot Bioconjugates for Ultrasensitive Nonisotopic Detection. Adv. Sci. 1998, 281, 20162018.

(31) Algar, W. R.; Krull, U. J. Luminescence and Stability of Aqueous Thioalkyl Acid Capped CdSe/ZnS Quantum Dots Correlated to Ligand Ionization. ChemPhysChem 2007, 8, 561-568.

(32) Aldana, J.; Wang, Y. A.; Peng, X. Photochemical Instability of CdSe Nanocrystals Coated by Hydrophilic Thiols. J. Am. Chem. Soc. 2001, 123, 8844-8850.

(33) Ben-Shahar, Y.; Scotognella, F.; Waiskopf, N.; Kriegel, I.; Dal Conte, S.; Cerullo, G.; Banin, U. Effect of Surface Coating on the Photocatalytic Function of Hybrid CdS-Au Nanorods. Small 2015, 11, 462-471.

(34) Xia, C.; Meeldijk, J. D.; Gerritsen, H. C.; de Mello Donega, C. Highly Luminescent Water-Dispersible NIR-Emitting Wurtzite $\mathrm{CuInS}_{2} / \mathrm{ZnS}$ Core/Shell Colloidal Quantum Dots. Chem. Mater. 2017, 29, 4940-4951.

(35) Zhao, C.; Bai, Z.; Liu, X.; Zhang, Y.; Zou, B.; Zhong, H. Small GSH-Capped CuInS ${ }_{2}$ Quantum Dots: MPA-Assisted Aqueous Phase Transfer and Bioimaging Applications. ACS Appl. Mater. Interfaces 2015, 7, 17623-17629.

(36) Tamang, S.; Beaune, G.; Texier, I.; Reiss, P. Aqueous Phase Transfer of InP/ZnS Nanocrystals Conserving Fluorescence and High Colloidal Stability. ACS Nano 2011, 5, 9392-9402.

(37) Nann, T. Phase-Transfer of CdSe@ZnS Quantum Dots Using Amphiphilic Hyperbranched Polyethylenimine. Chem. Commun. 2005, 1735-1736.

(38) Ben-Shahar, Y.; Scotognella, F.; Waiskopf, N.; Kriegel, I.; Dal Conte, S.; Cerullo, G.; Banin, U. Effect of Surface Coating on the Photocatalytic Function of Hybrid CdS-Au Nanorods. Small 2015, 11, 462-471.

(39) Lees, E. E.; Nguyen, T. L.; Clayton, A. H. A.; Mulvaney, P. The Preparation of Colloidally Stable, Water-Soluble, Biocompatible, 
Semiconductor Nanocrystals with a Small Hydrodynamic Diameter. ACS Nano 2009, 3, 1121-1128.

(40) Turo, M. J.; Macdonald, J. E. Crystal-Bound vs Surface-Bound Thiols on Nanocrystals. ACS Nano 2014, 8, 10205-10213.

(41) Chen, L.; Li, G. Functions of 1-Dodecanethiol in the Synthesis and Post-Treatment of Copper Sulfide Nanoparticles Relevant to Their Photocatalytic Applications. ACS Appl. Nano Mater. 2018, 1, 4587-4593.

(42) Xie, R.; Rutherford, M.; Peng, X. Formation of High-Quality IIII-VI Semiconductor Nanocrystals by Tuning Relative Reactivity of Cationic Precursors. J. Am. Chem. Soc. 2009, 131, 5691-5697.

(43) So, D.; Konstantatos, G. Thiol-Free Synthesized Copper Indium Sulfide Nanocrystals as Optoelectronic Quantum Dot Solids. Chem. Mater. 2015, 27, 8424-8432.

(44) Pan, Z.; Mora-Sero, I.; Shen, Q.; Zhang, H.; Li, Y.; Zhao, K.; Wang, J.; Zhong, X.; Bisquert, J. High-Efficiency Green Quantum Dot Solar Cells. J. Am. Chem. Soc. 2014, 136, 9203-9210.

(45) Akdas, T.; Distaso, M.; Kuhri, S.; Winter, B.; Birajdar, B.; Spiecker, E.; Guldi, D. M.; Peukert, W. The Effects of Post-Processing on the Surface and the Optical Properties of Copper Indium Sulfide Quantum Dots. J. Colloid Interface Sci. 2015, 445, 337-347.

(46) Gromova, M.; Lefrancois, A.; Vaure, L.; Agnese, F.; Aldakov, D.; Maurice, A.; Djurado, D.; Lebrun, C.; de Geyer, A.; Schulli, T. U.; et al. Growth Mechanism and Surface State of $\mathrm{CuInS}_{2}$ Nanocrystals Synthesized with Dodecanethiol. J. Am. Chem. Soc. 2017, 139, $15748-15759$.

(47) Robinson, E. H.; Turo, M. J.; Macdonald, J. E. Controlled Surface Chemistry for the Directed Attachment of Copper(I) Sulfide Nanocrystals. Chem. Mater. 2017, 29, 3854-3857.

(48) Wang, Y.; Hu, Y.; Zhang, Q.; Ge, J.; Lu, Z.; Hou, Y.; Yin, Y. One-Pot Synthesis and Optical Property of Copper(I) Sulfide Nanodisks. Inorg. Chem. 2010, 49, 6601-6608.

(49) Van Der Stam, W.; Berends, A. C.; Rabouw, F. T.; Willhammar, T.; Ke, X.; Meeldijk, J. D.; Bals, S.; De Mello Donega, C. Luminescent $\mathrm{CuInS}_{2}$ Quantum Dots by Partial Cation Exchange in $\mathrm{Cu}_{2-\mathrm{X}} \mathrm{S}$ Nanocrystals. Chem. Mater. 2015, 27, 621-628.

(50) Wen, X.; Zhang, W.; Yang, S.; Dai, Z. R.; Wang, Z. L. Solution Phase Synthesis of $\mathrm{Cu}(\mathrm{OH})_{2}$ Nanoribbons by Coordination SelfAssembly Using $\mathrm{Cu}_{2} \mathrm{~S}$ Nanowires as Precursors. Nano Lett. 2002, 2, 1397-1401.

(51) Trevani, L. N.; Roberts, J. C.; Tremaine, P. R. Copper(II)Ammonia Complexation Equilibria in Aqueous Solutions at Temperatures from 30 to 250 Degrees C by Visible Spectroscopy. J. Solution Chem. 2001, 30, 585-622.

(52) Lamble, G.; Moen, A.; Nicholson, D. G. Structure of the Diamminecopper(1) Ion in Solution An X-Ray Absorption Spectroscopic Study. J. Chem. Soc., Faraday Trans. 1994, 90 (15), 2211.

(53) Sano, M.; Maruo, T.; Yamatera, H. Structural Determination of Solvated Copper Species in Formamide Solution by EXAFS. J. Chem. Phys. 1988, 89, 1185-1187.

(54) Nisar Ansari, M.; Khan, S. H.; Aziz Khan, A. Polarographic Studies on Copper(II)-Formamide Complex in Alkaline Medium. J. Electroanal. Chem. Interfacial Electrochem. 1970, 28, 450-452.

(55) Xu, J.; Xue, D.; Zhu, Y. Room Temperature Synthesis of Curved Ammonium Copper Molybdate Nanoflake and Its Hierarchical Architecture. J. Phys. Chem. B 2006, 110, 17400-17405.

(56) Zhang, Z.; Shao, X.; Yu, H.; Wang, Y.; Han, M. Morphosynthesis and Ornamentation of 3D Dendritic Nanoarchitectures. Chem. Mater. 2005, 17, 332-336.

(57) Lou, J. L.; Shiu, H. W.; Chang, L. Y.; Wu, C. P.; Soo, Y.-L.; Chen, C.-H. Preparation and Characterization of an Ordered 1Dodecanethiol Monolayer on Bare Si(111). Langmuir 2011, 27, 3436-3441.

(58) Xie, Y.; Riedinger, A.; Prato, M.; Casu, A.; Genovese, A.; Guardia, P.; Sottini, S.; Sangregorio, C.; Miszta, K.; Ghosh, S.; et al. Copper Sulfide Nanocrystals with Tunable Composition by Reduction of Covellite Nanocrystals with $\mathrm{Cu}^{+}$Ions. J. Am. Chem. Soc. 2013, 135, 17630-17637.
(59) Kriegel, I.; Jiang, C.; Rodríguez-Fernández, J.; Schaller, R. D.; Talapin, D. V.; da Como, E.; Feldmann, J. Tuning the Excitonic and Plasmonic Properties of Copper Chalcogenide Nanocrystals. J. Am. Chem. Soc. 2012, 134, 1583-1590.

(60) Hinterwirth, H.; Kappel, S.; Waitz, T.; Prohaska, T.; Lindner, W.; Lämmerhofer, M. Quantifying Thiol Ligand Density of SelfAssembled Monolayers on Gold Nanoparticles by Inductively Coupled Plasma-Mass Spectrometry. ACS Nano 2013, 7, 1129-1136.

(61) Aldana, J.; Lavelle, N.; Wang, Y.; Peng, X. Size-Dependent Dissociation $\mathrm{pH}$ of Thiolate Ligands from Cadmium Chalcogenide Nanocrystals. J. Am. Chem. Soc. 2005, 127, 2496-2504.

(62) Schrier, J.; Wang, L. W. On the Size-Dependent Behavior of Nanocrystal-Ligand Bonds. J. Phys. Chem. B 2006, 110, 1198211985.

(63) Asgary, S.; Mirabbaszadeh, K.; Nayebi, P.; Emadi, H. Synthesis and Investigation of Optical Properties of TOPO-Capped CuInS Semiconductor Nanocrystal in the Presence of Different Solvent. Mater. Res. Bull. 2014, 51, 411-417.

(64) Kruszynska, M.; Borchert, H.; Parisi, J.; Kolny-Olesiak, J. Investigations of Solvents and Various Sulfur Sources Influence on the Shape-Controlled Synthesis of $\mathrm{CuInS}_{2}$ Nanocrystals. J. Nanopart. Res. 2011, 13, 5815-5824.

(65) Kruszynska, M.; Borchert, H.; Bachmatiuk, A.; Rümmeli, M. H.; Büchner, B.; Parisi, J.; Kolny-Olesiak, J. Size and Shape Control of Colloidal Copper(I) Sulfide Nanorods. ACS Nano 2012, 6, 58895896.

(66) Hens, Z.; Martins, J. C. A Solution NMR Toolbox for Characterizing the Surface Chemistry of Colloidal Nanocrystals. Chem. Mater. 2013, 25, 1211-1221.

(67) Owen, J. S.; Park, J.; Trudeau, P. E.; Alivisatos, A. P. Reaction Chemistry and Ligand Exchange at Cadmium-Selenide Nanocrystal Surfaces. J. Am. Chem. Soc. 2008, 130, 12279-12281.

(68) Kopping, J. T.; Patten, T. E. Identification of Acidic Phosphorus-Containing Ligands Involved in the Surface Chemistry of CdSe Nanoparticles Prepared in Tri-N-Octylphosphine Oxide Solvents. J. Am. Chem. Soc. 2008, 130, 5689-5698.

(69) Becerra, L. R.; Murray, C. B.; Griffin, R. G.; Bawendi, M. G. Investigation of the Surface Morphology of Capped CdSe Nanocrystallites by ${ }^{31} \mathrm{P}$ Nuclear Magnetic Resonance. J. Chem. Phys. 1994, 100, 3297-3300.

(70) De Roo, J.; Yazdani, N.; Drijvers, E.; Lauria, A.; Maes, J.; Owen, J. S.; Van Driessche, I.; Niederberger, M.; Wood, V.; Martins, J. C.; et al. Probing Solvent-Ligand Interactions in Colloidal Nanocrystals by the NMR Line Broadening. Chem. Mater. 2018, 30, 5485-5492.

(71) Berends, A. C.; Van Der Stam, W.; Hofmann, J. P.; Bladt, E.; Meeldijk, J. D.; Bals, S.; De Mello Donega, C. Interplay between Surface Chemistry, Precursor Reactivity, and Temperature Determines Outcome of $\mathrm{ZnS}$ Shelling Reactions on $\mathrm{CuInS}_{2}$ Nanocrystals. Chem. Mater. 2018, 30, 2400-2413.

(72) Watts, J. F.; Leadley, S. R.; Castle, J. E.; Blomfield, C. J. Adsorption of PMMA on Oxidized $\mathrm{Al}$ and Si Substrates: An Investigation by High-Resolution X-Ray Photoelectron Spectroscopy. Langmuir 2000, 16, 2292-2300.

(73) Wilson, D.; Langell, M. A. XPS Analysis of Oleylamine/Oleic Acid Capped $\mathrm{Fe}_{3} \mathrm{O}_{4}$ Nanoparticles as a Function of Temperature. Appl. Surf. Sci. 2014, 303, 6-13.

(74) Zhang, L.; He, R.; Gu, H. C. Oleic Acid Coating on the Monodisperse Magnetite Nanoparticles. Appl. Surf. Sci. 2006, 253, 2611-2617.

(75) Wagener, K.; Batich, C.; Kirsch, B.; Wanigatunga, S. Chain Propagation/Step Propagation Polymerization. III. An XPS Investigation of Poly(Oxyethylene)-b-Poly(Pivalolactone) Telechelomer. J. Polym. Sci., Part A: Polym. Chem. 1989, 27, 2625-2631.

(76) Williamson, C. B.; Nevers, D. R.; Hanrath, T.; Robinson, R. D. Prodigious Effects of Concentration Intensification on Nanoparticle Synthesis: A High-Quality, Scalable Approach. J. Am. Chem. Soc. 2015, 137, 15843-15851. 